\title{
RIESZ POINTS OF THE SPECTRUM OF AN ELEMENT IN A SEMISIMPLE BANACH ALGEBRA( ${ }^{(1)}$
}

BY

\section{LYNN D. PEARLMAN}

\begin{abstract}
Let $A$ be a semisimple Banach algebra with unit element and let $S_{A}$ denote the socle of $A$. For an element $y$ in $A$, let $L_{y}\left[R_{y}\right]$ denote the operator of left [right] multiplication by $y$ on $A$. The operational calculus and A. E. Taylor's theory of the ascent $a(T)$ and descent $\delta(T)$ of an operator $T$ on $A$ are used to show that the following conditions on a number $\lambda$ in the spectrum of an element $x$ in $A$ are all equivalent. (1) $\lambda$ is a pole of the resolvent mapping $z \rightarrow(z-x)^{-1}$ and the spectral idempotent $f$, for $x$ at $\lambda$ is in $S_{A^{*}}$ (2) $\lambda-x-c$ is invertible in $A$ for some $c$ in the closure of $S_{A}$ such that $c x=x c$. (3) $\lambda-x$ is invertible modulo the closure of $S_{A}$ and $0<a\left(L_{(\lambda-x)}\right)=\delta\left(L_{(\lambda-x)}\right)<\infty$. (4) $\lambda-x$ is invertible modulo the closure of $S_{A}$ and $0<a\left(R_{(\lambda-x)}\right)=\delta\left(R_{(\lambda-x)}\right)=a(L(\lambda-x)=\delta(L(\lambda-x)$ $<\infty$. Such numbers $\lambda$ are called Riesz points. An element $x$ is called a Riesz element of $A$ if it is topologically nilpotent modulo the closure of $S_{A}$. It is shown that $x$ is a Riesz element if and only if every nonzero number in the spectrum of $x$ is a Riesz point.
\end{abstract}

Introduction. Previous authors ([4], [14], [7]) have studied poles of the resolvent with finite rank for operators on Banach space. Many of their results are generalized here to study the spectra of elements in a semisimple Banach algebra A.

In $\$ 1$, the generalized Fredholm theory [3] is outlined. It is then used to give a necessary and sufficient condition for an arbitrary element $x$ in $A$ to have an associated element $s$ in the socle such that $x+s$ is invertible. In $\$ 2$, numbers which are poles of the resolvent mapping are considered. The theorems from the first two sections are used in $\$ 3$ to give several characterizations of Riesz points of the spectrum of an element. Finally in $\$ 4$ the Riesz elements of $A$, the analogous to well-known Riesz operators on Banach space, are considered.

Presented to the Society, January 26, 1973 under the title Riesz points of the spectrum; received by the editors February 23, 1973.

AMS (MOS) subject classifications (1970). Primary 46H05; Secondary 47B30, 47A55.

Key words and phrases. Semisimple Banach algebra, Fredholm element socle, ascent, descent, Riesz operator.

(1) Some of the results of this work form a portion of the author's doctoral dissertation directed by B. A. Barnes at the University of Oregon, 1972.

Copyright $\odot$ 1974, American Mathematical Society 
1. The generalized Fredholm theory. Let $A$ be a semisimple Banach algebra with unit element 1 . The socle of $A$ is denoted by $S_{A}$ and the elements of the socle are called finite. Let $\pi: A \rightarrow A / \bar{S}$ be the quotient homomorphism and let $J_{A}$ denoce the Jacobson radical of the Banach algebra $A / \bar{S}_{A}$. The pre-image of $J_{A}$ in $A, \pi^{-1}\left(J_{A}\right)$, is the intersection of all the primitive ideals of $A$ containing $S_{A}$. This closed ideal is denoted by $I_{A}$ and is called the ideal of inessential elements. The open semigroup $\Phi_{A}=\left\{x \in A: \pi(x)\right.$ is invertible in $\left.A / \bar{S}_{A}\right\}$ is called the set of Fredbolm elements. For each two-sided ideal $M$ of $A$ such that $S_{A} \subseteq$ $M \subseteq I_{A}, \Phi_{A}=\{x \in A: x+M$ is invertible in $A / M\}$ [3]. Then, for all $x$ in $\Phi_{A}$ and $y$ in $I_{A^{\prime}} x$ and $x+y$ are in the same component of $\Phi_{A}$. The set of minimal idempotents of $A$ is denoted by $E_{A}$. Of course $E_{A}$ may be empty, in which case $S_{A}=$ \{0\}. The subscripts will be omitted when the a lgebra $A$ is understood.

For any subset $K$ of $A$, let $L[K]=\{x \in A: x K=\{0\}\}$ and $R[K]=\{x \in A$ : $K x=\{0\}\}$. The set $L[K](R[K])$ is a closed left (right) ideal of $A$. Since $A$ has no nonzero nilpotent left or right ideals, $L[A]=\{0\}=R[A]$. Hence, for each $x$ in $A$, $L[x A]=\{y: y x=0\}$ and $R[A x]=\{y: x y=0\}$.

Let $K$ be a right (left) ideal of $A$ contained in $S$. Any maximal orthogonal set of minimal idempotents in $K$ has the same cardinality denoted by $\theta(K)$ and called the order of $K$. If $n=\theta(K)$ is finite and $\left\{e_{1}, e_{2}, \cdots, e_{n}\right\}$ is a maximal orthogonal set of minimal idempotents contained in $K$, then

$$
\sum_{i=1}^{n} e_{i} A=K \quad\left(\sum_{i=1}^{n} A e_{i}=K\right) .
$$

A right (left) ideal $K$ has finite order $n$ if and only if $K$ is the direct sum of $n$ minimal right (left) ideals of $A$. If $e$ is an idempotent in $S, \theta(A e)=\theta(e A)<\infty$. Let $\theta(e)$ be this integer. For proofs see [2, 2$]$. The following crucial theorem was proved by Barnes in [3].

1.1. Theorem. An element $x$ of $A$ is a Fredbolm element if and only if there are idempotents $e$ and $f$ in $S$ such that

$$
(1-f) A=x A, \quad A(1-e)=A x, \quad A=A x \oplus A e, \quad A=x A \oplus f A .
$$

For each $x$ in $\Phi$ let $k(x)=\theta(L[x A])-\theta(R[A x])$. The integer $k(x)$ is called the generalized Fredbolm index of $x$. The mapping $x \mapsto k(x)$ is a continuous function on $\Phi[3$, Theorem 4.1]. Since $k$ is integer valued, it is constant on components of $\Phi$. Hence, for $x$ in $\Phi$ and $y$ in $l, k(x+y)=k(x)$. Another important property of the generalized Fredholm index is that it acts as a semigroup homomorphis $m$ of $\Phi$ into the integers, that is $k(x y)=k(x)+k(y)$ for all $x$ and $y$ in $\Phi[3, \S 3]$.

Let $X$ be a Banach space and let $B(X)$ denote the primitive algebra of bounded operators on $X$. The socle of $B(X)$ is the ideal $\mathcal{F}(X)$ of operators with finite- 
dimensional range. The closed ideal of compact operators contains $\mathcal{F}(X)$ and is contained in the ideal of inessential operators $([8, \mathrm{pp} .278-283]$ and $[6$, Theorem 1]). The operators $T$ with closed range, finite nullity $n(T)$, and finite defect $d(T)$ are usually called Fredholm operators. They coincide with the semigroup of Fredholm elements of $B(X)$. In [3, p. 91], Barnes shows that, for a Fredholm operator $T, k(T)=d(T)-n(T)$ which is the usual index of $T$ in Fredholm operator theory.

Schechter shows in his paper [11] that if $T$ is a Fredholm operator of index zero there is an operator $U$ in $\mathcal{F}(X)$ such that $T+U$ is invertible. Examples will be given at the end of this section to show that there are nonprimitive semisimple Banach algebras $A$ containing Fredholm elements $x$ of index zero such that $x+u$ is singular for all $u$ in the socle of $A$. It is natural to ask: "Which elements of $\Phi_{A}$ can be carried into the invertible elements by adding an element of $S$ ?" To answer this question some facts about primitive ideals of $A$ are needed.

Let $Q$ be a primitive ideal of $A$. Since $Q$ is closed, $A / Q$ is a primitive Banach algebra with unit element. Hence $S_{A / Q}$ and $\Phi_{A / Q}$ exist. Let $k_{A / Q}$ denote the generalized Fredholm index defined on $\Phi_{A / Q}$ and let $\zeta_{Q}: A \rightarrow A / Q$ be the quotient homomorphism. For each $f$ in $E, \zeta_{Q}(f)$ is an idempotent and, if $f$ is not in $Q, \zeta_{Q}(f)$ is in $E_{A / Q}$ (the set of minimal idempotents of the algebra $A / Q)$ since $f A f$ is one dimensional. Then clearly $\zeta_{Q}\left(S_{A}\right) \subseteq S_{A / Q}$ and, since $\zeta_{Q}$ is continuous, $\zeta_{Q}\left(\bar{S}_{A}\right) \subseteq \bar{S}_{A / Q}$, where $S_{A}$ and $S_{A / Q}$ denote the socles of $A$ and $A / Q$ respectively. Hence $\zeta_{Q}\left(\Phi_{A}\right) \subseteq \Phi_{A} / Q$. Let $I_{A / Q}$ be the ideal of inessential elements of $A / Q$. Let $\pi_{Q}: \zeta_{Q}(A) \rightarrow \zeta_{Q}(A) / \bar{S}_{\zeta_{Q}(A)}$ be the quotient homomorphism. Then $\pi_{Q}\left(\zeta_{Q}\left(I_{A}\right)\right)$ is a quasiregular ideal in $\pi_{Q}\left(\zeta_{Q}(A)\right)$ because $\zeta_{Q}\left(\bar{S}_{A}\right) \subseteq \bar{S}_{\zeta_{Q}(A)}$ and so $\zeta_{Q}\left(I_{A}\right)$ is contained in $I_{A / Q}$. For any minimal idempotent $e$ in $E$ define $L^{e}=\{x \in A: x A \subseteq A(1-e)\}$ and $R^{e}=\{x \in A: A x \subseteq(1-e) A\}$. These two ideals are primitive and in [1] Barnes proves that

$$
L^{e}=R^{e}=L[A e]=R[e A]
$$

1.2. Lemma. If $Q$ is a primitive ideal of $A$ then either $S$ is contained in $Q$ or $Q=L^{e}$ for each $e$ in $E \backslash Q$.

Proof. Suppose $Q$ is a primitive ideal and $S$ is not contained in $Q$. Let $e$ be a minimal idempotent which is not in $Q$. Since $L\left[L^{e}\right] L^{e}=\{0\} \subseteq Q$, either $L\left[L^{e}\right]$ $\subseteq Q$ or $L^{e} \subseteq Q$ by $[9,2.2 .9]$. Now $e$ is in $L\left[L^{e}\right]$ but not in $Q$ so $Q$ contains $L^{e}$. Also $e Q$ is a right ideal not containing $e$ so $e Q \neq e A$, and since $e A$ is a minimal right ideal this implies $e Q=\{0\}$. Then $e A Q \subseteq e Q=\{0\}$ so $Q$ is contained in $R[e A]=L^{e}$.

1.3. Definition. Let $\psi=\left\{x \in \Phi: k_{A} / Q\left(\zeta_{Q}(x)\right)=0\right.$ for all primitive ideals $\left.Q\right\}$. 
For each $e$ in $E$ abbreviate and write $\zeta_{e}$ for $\zeta_{L} e$ and let $k_{e}$ denote the generalized Fredholm index on $\Phi_{\zeta_{e}(A)}$. Using Lemma 1.2 it is clear that $\psi=\{x \in A$ : $k_{e}\left(\zeta_{e}(x)\right)=0$ for all $\left.e \in E\right\}$. Let $\psi_{n}=\{x \in \psi: n=\theta(R[A x])\}$ for each integer $n$. Then $\psi$ is the union of the sets $\psi_{n}$.

1.4. Theorem. For each $x$ in $\psi$ there is an $s$ in $S$ such that $x+s$ is invertible. In particular $s$ can be chosen to be in fAe for finite idempotents $e$ and $f$ such that $L[x A]=$ Af and $R[A x]=e A$.

Proof. Let $x$ be in $\psi_{0}$, then $R[A x]=\{0\}$. By Theorem 1.1, $A=A x$. If also $L[x A]=\{0\}$ then, by $[9,1.6 .9], x$ is invertible. Choosing $s=0$ would satisfy the requirements of the theorem. Suppose $L[x A] \neq\{0\}$. Let $\left\{f_{1}, f_{2}, \cdots, f_{n}\right\}$ be a maximal orthogonal set of minimal idempotents in $L[x A]$. Since $f_{1}$ is not in $L^{f_{1}}$, and $\zeta_{f_{1}}\left(f_{1}\right) \zeta_{f_{1}}(x)=\zeta_{f_{1}}(0)$ it follows that the left ideal $\left\{\zeta_{f_{1}}(y): \zeta_{f_{1}}(y x)=\zeta_{f_{1}}(0)\right\}=$ $L\left[\zeta_{f_{1}}(x)\right]$ has nonzero order. The right annihilator of the primitive algebra $\zeta_{f_{1}}(A)$ is zero and, since $A x=A, \theta\left(R\left[\zeta_{f_{1}}(A x)\right]\right)=0$. Then

$$
k_{f_{1}}\left(\zeta_{f_{1}}(x)\right)=\theta\left(L\left[\zeta_{f_{1}}(x A)\right]\right)-\theta\left(R\left[\zeta_{f_{1}}(A x)\right]\right) \neq 0,
$$

a contradiction. Hence $\theta(L[x A])=\theta(R[A x])=0$ for all $x$ in $\psi_{0}$.

Now suppose $q$ is a nonnegative integer and for all $0 \leq n \leq q$ the theorem is true for all elements $x$ of $\psi_{n}$. Let $x$ be in $\psi_{q+1}$. Let $\left\{e_{1}, e_{2}, \ldots, e_{q+1}\right\}$ be a maximal orthogonal set of minimal idempotents contained in $R[A x]$. Let $\left\{f_{1}\right.$, $\left.f_{2}, \cdots, f_{m}\right\}$ be a maximal orthogonal set of minimal idempotents in $L[x A]$ for some integer $m \geq 0$. Since $e_{1}$ is not in $L^{e_{1}}, \zeta_{e_{1}}\left(e_{1}\right)$ is a nonzero element of $R\left[\zeta_{e_{1}}(A x)\right]$ so this right ideal has positive order. Let $f=\sum_{j=1}^{m} f_{j}$. Assume that $f_{j} A e_{1}=\{0\}$ for all $0 \leq j \leq m$. Then $f A e_{1}=\{0\}$ and $\zeta_{e_{1}}(1-f)=\zeta_{e_{1}}(1)$. By Theorem 1.1, $(1-f) A=x A$ and it follows that $\zeta_{e_{1}}(A)=\zeta_{e_{1}}(x A)$. Since $\zeta_{e_{1}}(A)$ is primitive, $\left\{\zeta_{e_{1}}(0)\right\}=L\left[\zeta_{e_{1}}(x A)\right]$, but this implies

$$
k_{e_{1}}\left(\zeta_{e_{1}}(x)\right)=\theta\left(L\left[\zeta_{e_{1}}(x A)\right]\right)-\theta\left(R\left[\zeta_{e_{1}}(A x)\right]\right) \neq 0,
$$

contradicting the fact that $x$ is in $\psi$. There must be some $1 \leq j \leq m$ such that $f_{j} A e_{1} \neq\{0\}$. Reorder the $\left\{f_{j}\right\}_{j=1}^{m}$, if necessary, so that $f_{1} A e_{1} \neq\{0\}$.

Let $t_{1}$ be an element of $A$ such that $f_{1} t_{1} e_{1} \neq 0$. Let $x_{1}=x+f_{1} t_{1} e_{1}$. Clearly $x_{1}$ is in $\Phi$. For each primitive ideal $Q$ of $A, \zeta_{Q}\left(f_{1} t_{1} e_{1}\right)$ is a finite element of $A / Q$ so

$$
\begin{aligned}
k_{A / Q}\left(\zeta_{Q}\left(x_{1}\right)\right) & =k_{A / Q}\left(\zeta_{Q}(x)+\zeta_{Q}\left(f_{1} t_{1} e_{1}\right)\right) \\
& =k_{A / Q}\left(\zeta_{Q}(x)\right)=0 .
\end{aligned}
$$

Then $x_{1}$ is in $\psi$. 
Now we will show that $x_{1}$ is in $\psi_{q}$ by showing that $R\left[A x_{1}\right]=\sum_{i=2}^{q+1} e_{i} A$. Let $y$ be in $R\left[A x_{1}\right]$, then $x y=-f_{1} t_{1} e_{1} y$. Multiplying by $f_{1}$ on the left we have $0=$ $f_{1} x y=-f_{1} t_{1} e_{1} y=x y$, so at least it is known that $y=\sum_{i=1}^{q+1} e_{i} y$. Since $e_{1}$ is a minimal idempotent and $f_{1} t_{1} e_{1} \neq 0, A f_{1} t_{1} e_{1}=A e_{1}$. Then $e_{1} y=0$ because $\{0\}=$ $A f_{1} t_{1} e_{1} y=A e_{1} y$. This shows that $R\left[A x_{1}\right]$ is contained in $\sum_{i=2}^{q+1} e_{1} y$. The reverse inclusion is obvious. Similarly it can be shown that $L\left[x_{1} A\right]=\sum_{j=2}^{m} A f_{j}$.

Since $x_{1}$ is in $\psi_{q}$, we know that $m-1=q$ and there is an $s$ in $\left(\sum_{j=2}^{q+1} f_{j}\right) A\left(\sum_{i=2}^{q+1} e_{i}\right)$ such that $x_{1}+s$ is invertible by the inductive assumption. Then $f_{1} t_{1} e_{1}+s$ is in $\left(\sum_{j=1}^{q+1} f_{j}\right) A\left(\sum_{i=1}^{q+1} e_{1}\right)$ and $x+\left(f_{1} t_{1} e_{1}+s\right)=x_{1}+s$ is invertible.

1.5. Corollary. Let $N$ be an ideal of $A$ such that $S \subset N \subset I$. An element $x$ of $A$ is in $\psi$ if and only if there is an element $z$ in $N$ such that $x+z$ is invertible.

Proof. It is sufficient to show that if there is a $z$ in $N$ such that $x+z$ is invertible then $x$ is in $\psi$. Let $Q$ be any primitive ideal of $A$. Since $\zeta_{Q}(z)$ is in $I_{A / Q}$, as discussed earlier, it follows that

$$
k_{Q}\left(\zeta_{Q}(x)\right)=k_{Q}\left(\zeta_{Q}(x)+\zeta_{Q}(z)\right)=0 .
$$

Therefore $x$ is in $\psi$.

1.6. Corollary. If $A$ is a primitive Banach algebra with unit element, $\psi=$ $\{x \in \Phi: k(x)=0\}$.

Proof. By Lemma 1.2, $\{0\}=L^{e}$ for all minimal idempotents $e$ and every nonzero primitive ideal of $A$ contains $S$. Then if $Q$ is any nonzero primitive ideal of $A$ and $x$ is in $\Phi, x+Q$ is invertible in $A / Q$ and so $k_{Q}(x+Q)=0$.

The following two examples were developed in [3]. They are used here to illustrate the fact that Corollary 1.6 is not necessarily true if $A$ is not primitive.

1.7. Example. Let $\Delta$ be a compact Hausdorff space and let $\mathrm{X}$ be a Banach space. Let $A$ be the algebra of continuous functions defined on $\Delta$ with values in $B(X)$. The norm of an element $f$ in $A$ is defined by

$$
|f|=\operatorname{Sup}_{y \in \Delta}\|f(y)\|
$$

if $\|\cdot\|$ denotes the operator norm on $B(X)$. Then $A$ is a semis imple Banach algebra with unit element. An idempotent $e$ in $A$ is minimal if and only if there is an isolated point $y_{0}$ in $\Delta$ and a projection $E$ in $B(X)$ with one-dimensional range such that $e\left(y_{0}\right)=E$ and $e(y)=0$ for all $y \neq y_{0}$. It follows that $S_{A}$ is the set of all $f$ in $A$ such that $f$ takes the value zero at all but a finite set of isolated points of $\Delta$ and $f(y)$ is an operator with finite-dimensional range for all $y$ in $\Delta$. 
An element $b$ of $A$ is Fredholm if and only if $b(y)$ is a Fredholm operator for all $y$ in $\Delta$ and is invertible except at perhaps a finite number of isolated points of $\Delta$. For a Fredholm element $b$ of $A, k(b)=\Sigma_{y \in \Delta} k^{\prime}(b(y))$, where $k^{\prime}$ is the usual index on $\Phi_{B(X)^{\circ}}$

For an $e$ in $E_{A}$ let $y_{0}$ be an isolated point such that $e(y)=0$ if and only if $y \neq y_{0}$. Then $L^{e}=\left\{f \in A: f\left(y_{0}\right)=0\right\}$ and the mapping $f+L^{e} \mapsto f\left(y_{0}\right)$ is an algebra isomorphism of $A / L^{e}$ onto $B(X)$. If $b \in A$ is Fredholm of index zero modulo $L^{e}, b\left(y_{0}\right)$ is a Fredholm operator of index zero in $B(x)$. If $b$ is in $\psi, b(y)$ is invertible if $y$ is not isolated and $b(y)$ is a Fredholm operator of index zero at every point of $\Delta$.

1.8. Example. For a Banach space $X$ assume that $\eta$ is an index set and $\left\{X_{\mu}: \mu \in \eta\right\}$ is a collection of closed subspaces of $X$ with the following properties:

$$
\left.X_{a} \cap \overline{\left(\sum_{\mu \in \eta ; \mu \neq a} X_{\mu}\right.}\right)=\{0\} \text { for all } a \in \eta
$$

$$
\left.\overline{\left(\sum_{\mu \in \eta} X_{\mu}\right.}\right)=X
$$

We define $A$ to be the algebra of all operators $T$ in $B(X)$ such that $T$ is invariant on each $X_{\mu}$ for all $\mu \in \eta$. Then $A$ is a semisimple closed subalgebra of $B(X)$ containing the identity and $S_{A}=\mathcal{F}(X) \cap A$, where $\mathcal{F}(X)$ is the ideal of operators with finite-dimensional range. For each $\alpha \in \eta$ let $Y_{\alpha}$ be the set of bounded linear functionals $f_{a}$ on $X_{a}$ such that

$$
f_{\alpha}\left(X_{\mu}\right)=\{0\} \text { for all } \mu \neq \alpha \text {. }
$$

For any $0 \neq u_{a}$ in $X_{a}$ such that $f_{a}\left(u_{a}\right)=1$, let $E: X \rightarrow X$ be defined $E(x)=$ $f_{a}(x) u_{\alpha}$. Then $E$ is a minimal idempotent of $A$ and every minimal idempotent in $A$ arises in this way. An operator $U$ is in $S_{A}$ if and only if $U$ vanishes on all but finitely many of the subspaces $X_{\mu}$ and $U$ is in $\mathcal{F}(X)$. If $T$ is a Fredholm element there exist operators $R$ in $A$ and $U$ and $V$ in $S_{A}$ such that $T R=I-U$ and $R T=I-V$. Then the restriction of $T$ to any of the subspaces $X_{\mu}, T_{\mu}$, is a Fredholm operator on $X_{\mu}$ and $T_{\mu}$ is invertible on $X_{\mu}$ for all but perhaps finitely many $\mu$ in $\eta$. The generalized index of $T$ is given by $k(T)=\Sigma_{\mu \in \eta} k_{\mu}\left(T_{\mu}\right)$, where $k_{\mu}$ is the Fredholm index on $\Phi_{B\left(x_{\mu}\right)}$ for all $\mu$ in $\eta$. Let $E=f_{a}() u_{a}$ for some $a \in \eta$ be a minimal idempotent of $A$ as constructed above. The ideal $L^{E}=R[E A]$ $=L[A E]=\left\{T \in A: T\left(X_{\alpha}\right) \equiv 0\right\}$. Then the mapping $\left(T+L^{E}\right) \rightarrow T_{\alpha}$ is an algebra is omorphism of $A / L^{E}$ onto $B\left(X_{a}\right)$. If $T$ is Fredholm of index zero modulo $L^{E}$, then $T_{a}$ is a Fredholm operator of index zero in $B\left(X_{\alpha}\right)$. For each $T$ in $\psi_{A}, T_{\mu}$ is 
a Fredholm operator of index zero in $B\left(X_{\mu}\right)$ for all $\mu$ in $\eta$, and $T_{\mu}$ is invertible in $B\left(X_{\mu}\right)$ for all but perhaps finitely many $\mu$ in $\eta$.

1.9. Example. Let $l^{2}\left(\mathrm{Z}^{+}\right)$denote the square summable sequences. Let $H$ be the Hilbert space direct sum $H=l^{2}\left(\mathrm{Z}^{+}\right) \oplus l^{2}\left(\mathrm{Z}^{+}\right)$. Let $A$ be the subalgebra of $B(H)$ leaving each of the two direct summands invariant, constructed as in Example 1.8. For two operators $U$ and $W$ in $B\left(l^{2}\left(Z^{+}\right)\right)$define $U \oplus W\left(\left\{x_{i}\right\}_{i=1}^{\infty},\left\{y_{j}\right\}_{j=1}^{\infty}\right)$ $=\left(U\left\{x_{i}\right\}_{i=1}^{\infty}, W\left\{y_{j}\right\}_{j=1}^{\infty}\right)$. Then $U \oplus W$ is in $A$. Define $T, F$ and $P$ in $B\left(l^{2}\left(Z^{+}\right)\right)$ as follows:

$$
\begin{aligned}
& T\left(x_{1}, x_{2}, x_{3}, \ldots\right)=\left(0, x_{1}, x_{2}, x_{3}, \ldots\right), \\
& F\left(x_{1}, x_{2}, x_{3}, \ldots\right)=\left(x_{2}, x_{3}, \ldots\right), \\
& P\left(x_{1}, x_{2}, x_{3}, \ldots\right)=\left(x_{1}, 0,0, \ldots\right) .
\end{aligned}
$$

Let $I$ be the identity element of $B(H)$. Then

$$
(T \oplus F)(F \oplus T)=I-(P \oplus 0) \text { and }(F \oplus T)(T \oplus F)=I-(0 \oplus P) .
$$

The operators $(0 \oplus P)$ and $(P \oplus 0)$ are in $E_{A}$ and

$$
L[(T \oplus F) A]=A(P \oplus 0), \quad R[A(T \oplus F)]=(0 \oplus P) A .
$$

Hence $T \oplus F$ is in $\Phi_{A}$ and $k_{A}(T \oplus F)=1-1=0$. Suppose there is a $V$ in $S_{A}$ such that $(T \oplus F)-V$ is invertible in $A$. Let $V_{1}$ and $V_{2}$ be the restrictions of $V$ to the first and second coordinate spaces respectively. Then $V=V_{1} \oplus V_{2}$ and $V_{1}$ and $V_{2}$ are in $B\left(l^{2}\left(\mathrm{Z}^{+}\right)\right)$. Since $(T \oplus F)-V=\left(T-V_{1}\right) \oplus\left(F-V_{2}\right)$ is invertible, each of the operators $T-V_{1}$ and $T-V_{2}$ is invertible in $B\left(l^{2}\left(\mathrm{Z}^{\dagger}\right)\right.$ ). Clearly $V_{1}$ and $V_{2}$ are in the socle of $B\left(l^{2}\left(Z^{+}\right)\right)$. Since the index is invariant on components of $\Phi_{B\left(l^{2}\left(z^{+}\right)\right)}$,

$$
0=k_{B\left(l^{2}\left(\mathrm{Z}^{+}\right)\right)}\left(T-V_{1}\right)=k_{B\left(l^{2}\left(\mathrm{Z}^{+}\right)\right)}(T)=1
$$

and

$$
0=k_{B\left(l^{2}\left(\mathrm{Z}^{+}\right)\right)}\left(F-V_{2}\right)=k_{B\left(l^{2}\left(\mathrm{Z}^{+}\right)\right)}(F)=-1 .
$$

Hence $T \oplus F$ is an element of $\Phi_{A}$ with index zero which cannot be carried into the invertible elements by adding an element of $S_{A}$.

1.10. Example. Let $\Delta$ be a compact Hausdorff space conta ining exactly two isolated points $y_{1}$ and $y_{2}$. Let $H, T$ and $F$ be as in Example 1.9. Let $A$ be the algebra of continuous functions on $\Delta$ with values in $B(H)$ constructed in Example 1.7. Define

$$
f(y)=\left\{\begin{array}{ll}
T & \text { if } y=y_{1}, \\
F & \text { if } y=y_{2}, \\
l & \text { if } y \neq y_{i}
\end{array}(i=1,2) .\right.
$$


Clearly $f$ is in $\Phi_{A}$ and $k_{A}(f)=k^{\prime}(T)+k^{\prime}(F)=1-1=0$. Suppose there is an element $u$ in $S_{A}$ such that $f+u$ is invertible. Then $(f+u)(y)$ is invertible and $u(y)$ is in $\mathcal{F}\left(l^{2}\left(Z^{+}\right)\right)$for all $y$ in $\Delta$ and $u(y)=0$ if $y$ is not in $\left\{y_{1}, y_{2}\right\}$. Since $k$ is constant on components of $\Phi_{B\left(l^{2}\left(Z^{+}\right)\right)}$,

$$
0=k^{\prime}\left((f+u)\left(y_{1}\right)\right)=k^{\prime}\left(T+u\left(y_{1}\right)\right)=k^{\prime}(T)=1
$$

and

$$
0=k^{\prime}\left((f+u)\left(y_{2}\right)\right)=k^{\prime}\left(F+u\left(y_{2}\right)\right)=k^{\prime}(F)=-1 .
$$

We must conclude that no such element $u$ exists. Therefore $f$ is an element of $\Phi_{A}$ of index zero which cannot be carried into the invertible elements by adding a finite element.

2. Poles of the resolvent. Let $O$ be any open subset of $\mathbf{C}$ containing a compact set $K$. If $\gamma:[0,1] \rightarrow 0$ is a piecew ise continuous ly differentiable mapping, $\gamma$ is called an admissible cycle for $(O, K)$ if

(a) $\gamma[0,1]$ is contained in $O \backslash K$, and

(b) $W(y, z)=0$ if $z$ is not in 0 , and $W(\gamma, z)=1$ if $z$ is in $K$,

where $W(\gamma, z)$ is the winding number of $\gamma$ at $z$. For any $x$ in $A$ and $z$ a complex number not in the spectrum of $x, \mathrm{sp}(x)$, define $R_{z}(x)$ to be the inverse of $z-x$. The function $z \mapsto R_{z}(x)$, called the resolvent mapping for $x$, is analytic on $C \backslash s p(x)$. If $O$ is an open set containing $\mathrm{sp}(x)$ and $\gamma$ is an admissible cycle for $(0, \mathrm{sp}(x))$ define

$$
f_{\gamma}(x)=\frac{1}{2 \pi i} \int_{\gamma} f(z) R_{z}(x) d z,
$$

for each complex-valued function $f$ analytic on 0 . If $\gamma_{1}$ and $\gamma_{2}$ are both admissible cycles for $(O, \mathrm{sp}(x))$, one can use the general Cauchy theorem to show that $f_{\gamma_{1}}(x)=f_{\gamma_{2}}(x)$. We will write $f(x)$ to de note this element of $A$.

If $f$ and $g$ are complex-valued analytic functions in a neighborhood $U$ of $\mathrm{sp}(x)$ and $g$ is a complex-valued function analytic in an open set $V$ containing $\mathrm{sp}(f(x))$ then $(g \circ f)(x)=g(f(x))$. The proofs of these facts can be easily generalized from the usual operational calculus where $A$ is $B(X)$ for some Banach space $X$; see $[5]$.

2.1. A construction. Let $x$ be an element of $A$. In the special case that $\mathrm{sp}(x)$ contains an isolated point $\lambda$, we use the operational calculus to show necessary and sufficient conditions that $\lambda$ is a pole of the resolvent mapping $z \mapsto R_{z}(x)$. The following construction will be used repeatedly in our arguments.

Let $r$ be a positive number such that

$$
\{z:|z-\lambda| \leq 2 r\} \cap \operatorname{sp}(x)=\{\lambda\} .
$$

For each integer $m \leq-1$ let 


$$
f_{m}(z)= \begin{cases}0 & \text { if }|z-\lambda|>r \\ (z-\lambda)^{-(m+1)} & \text { if }|z-\lambda|<r\end{cases}
$$

For each integer $m \geq 0$ let

$$
f_{m}(z)= \begin{cases}(z-\lambda)^{-(m+1)} & \text { if }|z-\lambda|>r \\ 0 & \text { if }|z-\lambda|<r\end{cases}
$$

For all integers $m, f_{m}(z)$ is a function analytic in the open set

$$
U=\{z:|z-\lambda|<r \text { or }|z-\lambda|>r\}
$$

which contains $\operatorname{sp}(x)$. For any $z$ in $U$,

(a) $(z-\lambda) f_{m+1}(z)=f_{m}(z)$ for $m \geq 0$,

(b) $(z-\lambda) f_{m}(z)=f_{m-1}(z)$ for $m \leq-1$,

(c) $(z-\lambda) f_{0}(z)=1-f_{-1}(z)$,

(d) $(z-\lambda)^{-(m+1)} f_{-1}(z)=f_{m}(z)$ for $m \leq-1$.

Using the operational calculus defined above we have the following relations:

(a) $(x-\lambda) f_{m+1}(x)=f_{m}(x)$ for $m \geq 0$,

(b) $(x-\lambda) f_{m}(x)=f_{m-1}(x)$ for $m \leq-1$,

(c) $(x-\lambda) f_{0}(x)=1-f_{-1}(x)$,

(d) $(x-\lambda)^{-(m+1)} f_{-1}(x)=f_{m}(x)$ for $m \leq-1$.

(e) $f_{-1}(x)$ is a nonzero idempotent.

For $0<|z-\lambda|<r$ we have the Laurent expansion

$$
R_{z}(x)=\sum_{k=0}^{\infty}-(z-\lambda)^{k} f_{k}(x)+\sum_{k=1}^{\infty}(z-\lambda)^{-k} f_{-k}(x) ;
$$

see $\left[12\right.$, p. 305]. The nonzero idempotent $f_{-1}(x)$ is called the spectral idempotent for $x$ at $\lambda$. If $p$ is a positive integer and $f_{-p-1}(x)=0$ but $f_{-p}(x) \neq 0$, then $\lambda$ is called a pole of $R_{z}(x)$ of order $p$. If in addition, $f_{-1}(x)$ is a finite element with $\theta\left(f_{-1}(x)\right)=n<\infty$, then the pole $\lambda$ is said to have finite rank $n$.

For any bounded operator $T$ on $A$, define $\alpha(T)$, the ascent of $T$, to be the smallest nonnegative integer $n$ such that

$$
\left\{y \in A: T^{n+1} y=0\right\}=\left\{y \in A: T^{n} y=0\right\},
$$

or $+\infty$ if no such $n$ exists. Define $\delta(T)$, the descent of $T$, to be the smallest nonnegative integer $m$ such that

$$
\left\{T^{m+1} y: y \in A\right\}=\left\{T^{m} y: y \in A\right\},
$$

or $+\infty$ if no such $m$ exists. For any element $x$ in $A$ let $L_{x}$ and $R_{x}$ denote the left and right multiplication operators of $x$ on $A$, and define

$$
\begin{array}{ll}
\alpha_{r}(x)=\alpha\left(R_{x}\right), & \delta_{r}(x)=\delta\left(R_{x}\right), \\
\alpha_{l}(x)=\alpha\left(L_{x}\right), & \delta_{l}(x)=\delta\left(L_{x}\right) .
\end{array}
$$


2.2. Theorem (Taylor [13, Theorems 3.6 and 3.7]). Let $X$ be a Banacb space and let $T$ be in $B(X)$. If $\alpha(T)$ and $\delta(T)$ are both finite, then $\alpha(T)=\delta(T)$. Let $q=\alpha(T)=\delta(T)$. Then

$$
\left\{x \in X: T^{q} x=0\right\} \cap\left\{T^{q} y: y \in X\right\}=\{0\}
$$

and

$$
\left\{x \in X: T^{q} x=0\right\} \oplus\left\{T^{q} y: y \in X\right\}=X .
$$

Applying this theorem to the case of operators of left and right multiplication on a semisimple Barach algebra $A$ we have the following useful corollary.

2.3. Corollary. If $\alpha_{l}(x)$ and $\delta_{l}(x)$ are both finite, then they are equal and for $p=\alpha_{l}(x)=\delta_{l}(x)$ we bave

(a) $x^{p} A \cap R\left[A x^{p}\right]=\{0\}$ and $R\left[A x^{p}\right] \oplus x^{p} A=A$.

If $\alpha_{r}(x)$ and $\delta_{r}(x)$ are both finite, then they are equal and for $m=\alpha_{r}(x)=\delta_{r}(x)$ we have

(b) $A x^{m} \cap L\left[x^{m} A\right]=\{0\}$ and $L\left[x^{m} A\right] \oplus A x^{m}=A$.

2.4. Lemma. If $\lambda$ is a pole of $R_{z}(x)$ of order $p$ and if $f_{-1}(x)$ is the spectral idempotent for $x$ at $\lambda$, then

(a) $p=\alpha_{l}(\lambda-x)=\delta_{l}(\lambda-x)=\alpha_{r}(\lambda-x)=\delta_{r}(\lambda-x)$,

(b) $\left(1-f_{-1}(x)\right) A=(\lambda-x)^{p} A$,

(c) $A\left(1-f_{-1}(x)\right)=A(\lambda-x)^{p}$,

(d) $\{0\} \neq R\left[A(\lambda-x)^{p}\right]=f_{-1}(x) A$,

(e) $\{0\} \neq L\left[(\lambda-x)^{p} A\right]=A f_{-1}(x)$,

(f) $(\lambda-x)^{p} A$ and $A(\lambda-x)^{p}$ are closed,

(g) $(\lambda-x)^{p} A \oplus R\left[A(\lambda-x)^{p}\right]=A$,

(h) $A(\lambda-x)^{p} \oplus L\left[\left(\lambda-x^{p} A\right]=A\right.$.

Proof. For each integer $n$ construct $f_{n}(x)$ for $x$ at $\lambda$ as in (2.1). Since $f_{-1}(x)$ is an idempotent

$$
\begin{aligned}
& A=\left(1-f_{-1}(x)\right) A \oplus f_{-1}(x) A, \\
& A=A\left(1-f_{-1}(x)\right) \oplus A f_{-1}(x),
\end{aligned}
$$

and each of these one-sided ideals is closed. For each $n \geq p, 0=f_{-n-1}(x)=$ $(x-\lambda)^{n} f_{-1}(x)$ so we have $f_{-1}(x)$ contained in $R\left[A(\lambda-x)^{n}\right]$. If $y$ is in $R\left[A(\lambda-x)^{n}\right]$ for some positive integer $n$,

$$
0=f_{n-1}(x)(\lambda-x)^{n} y=(\lambda-x) f_{0}(x) y=\left(1-f_{-1}(x)\right) y \text {. }
$$

Hence $R\left[A(\lambda-x)^{n}\right]$ is contained in $f_{-1}(x) A$ for all positive integers $n$. Then $R\left[A(\lambda-x)^{n}\right]=f_{-1}(x) A$ for all $n \geq p$ and $\alpha_{r}(\lambda-x)=p$ since $0 \neq f_{-p}(x)=$ $(x-\lambda)^{p-1} f_{-1}(x)^{-1}$. Similarly, $L\left[(\lambda-x)^{n} A\right]=A f_{-1}(x)$ for all $n \geq p$ and $a_{l}(\lambda-x)=p$. This proves (d) and (e). 
To see that $\left(1-f_{-1}(x)\right) A$ is contained in $(\lambda-x)^{p} A$, compute $1-f_{-1}(x)=$ $(x-\lambda) f_{0}(x)=(x-\lambda)^{p} f_{p-1}^{-1}(x)$. If $z=(\lambda-x)^{p} y$ for some $y$ in $A$ then, using (1),

$$
z=\left(1-f_{-1}(x)\right)(\lambda-x)^{p} y+f_{-1}(x)(\lambda-x)^{p} y=\left(1-f_{-1}(x)\right) z .
$$

Therefore $\left(1-f_{-1}(x)\right) A=(\lambda-x)^{p} A$. Similarly $A(\lambda-x)^{p}=A\left(1-f_{-1}(x)\right)$. Now (b) and (c) are proved and they imply (f). Using (1), (b), and (d) we have (g), and using (2), (c) and (e) we have (h). Since

$$
(\lambda-x)^{2 p} A=(\lambda-x)^{p}\left(1-f_{-1}(x)\right) A=\left(1-f_{-1}(x)\right)^{2} A=(\lambda-x)^{p} A,
$$

we conclude $\delta_{l}(x) \leq p$. By Corollary $2.3, \delta_{l}(x)=p$. Similarly $\delta_{r}(x)=p$. This proves (a).

2.5. Definition. Let $n$ be a positive integer and $g$ a nonzero idempotent in $A$. We will say that an element $y$ of $A$ generates an $(n, g, R)$-decomposition of the algebra $A$ if

(a) $g y=y g$,

(b) $R\left[A y^{n}\right]=g A$, and

(c) $y^{n} A \oplus g A=A$.

Similarly, an element $y$ generates an $(n, g, L)$-decomposition of $A$ if

(d) $g y=y g$,

(e) $L\left[y^{n} A\right]=A g$, and

(f) $A y^{n} \oplus A g=A$.

2.6. Lemma. Let $x$ be in $A$ and let $\lambda$ be a complex number. Assume that $p$ is a positive integer, $g$ is a nonzero idempotent in $A,(\lambda-x)$ generates a $(p, g, R)$-decomposition of $A$ and $p$ is the smallest positive integer for which this is true. Then:

(a) $(\lambda-x)^{p} A=(1-g) A$,

(b) $p=a_{l}(\lambda-x)=\delta_{l}(\lambda-x)$,

(c) $\lambda$ is a pole of $R_{z}(x)$ of order $p$,

(d) $g$ is the spectral idempotent for $x$ at $\lambda$.

Proof. Clearly $(1-g)(\lambda-x)^{p}=(\lambda-x)^{p}=(\lambda-x)^{p}(1-g)$, and hence (a) is true. Then $(\lambda-x)^{p} A=(\lambda-x)^{p}(1-g) A=(\lambda-x)^{2 p} A$ and so we have $\delta_{l}(\lambda-x) \leq p$.

Assume that $(\lambda-x)^{p} z \neq 0$ but $(\lambda-x)^{p+1} z=0$. Let $y=(\lambda-x)^{p} z$. Then $(\lambda-x)^{p} y=0$ since $p \geq 1$. Hence $y$ is in $g A \cap(\lambda-x)^{p} A=\{0\}$, a contradiction.

This shows that $a_{l}(\lambda-x) \leq p$.

Let $B=(1-g) A(1-g)$. $B$ is a semisimple Banach algebra with unit element $(1-g)$. To see that $(\lambda-x)(1-g)$ is an invertible element in $B$, use $[9$, Theorem 1.6.9] and the two following computations:

and

$$
\begin{aligned}
B & =(\lambda-x)^{p} A(1-g)=(\lambda-x)^{p+1} A(1-g) \\
& =(\lambda-x)(1-g) A(1-g)=(\lambda-x) B
\end{aligned}
$$




$$
\begin{aligned}
R_{B}[B(\lambda-x)] & =\{y \in A:(1-g) y(1-g)=y \text { and } B(\lambda-x) y=\{0\}\} \\
& \subseteq\left\{y \in A: g y=0 \text { and }(1-g)(\lambda-x)^{p} y=0\right\} \\
& =\left\{y \in A: g y=0 \text { and }(\lambda-x)^{p} y=0\right\} \\
& =(1+g) A \cap g A=\{0\}
\end{aligned}
$$

where, for any subset $W$ of $B, R_{B}[W]=\{z \in B: W z=\{0\}\}$.

Since $0=(\lambda-x)^{p} g=((\lambda-x) g)^{p}$, the spectral radius in $A$ of the element $(\lambda-x) g$ is zero and hence $\operatorname{sp}_{A}\left((\lambda-x)_{g}\right)=\{0\}$. Since $g \neq 0,\{0\}=\operatorname{sp}_{A}\left((\lambda-x)_{g}\right)=$ $\{0\} \cup \mathrm{sp}((\lambda-x) g)[9, \mathrm{p} .35]$. Then $\mathrm{sp}_{g} A_{g}(x g)$ is just $\{\lambda\}$.

It is easy to check that $\operatorname{sp}_{A}(x)=\operatorname{sp}_{B}(x(1-g)) \cup s \mathrm{~s}_{g} A_{g}(x g)$. We know that $\mathrm{sP}_{B}((1-g) x)$ is compact and does not contain $\lambda$. Hence $\lambda$ is an isolated point of $\operatorname{sp}_{A}(x)$.

For each integer $m$, construct $f_{m}(x)$ at $\lambda$ as in 2.1. Let $z \mapsto P_{z}(x(1-g))$ be the resolvent mapping for $x(1-g)$ in the subalgebra $B$. For each $z \notin \operatorname{sp}_{B}(x(1-g))$,

$$
(1-g)=P_{z}(x(1-g))(z-x)(1-g)=(z-x)(1-g) P_{z}(x(1-g)) \text {. }
$$

Then, if $z$ is not in $\operatorname{sp}_{A}(x), P_{z}(x(1-g))=(1-g) R_{z}(x)=R_{z}(x)(1-g)$. The mapping $z \rightarrow P_{z}(x(1-g))$ is analytic on $\{z:|z-\lambda|<2 r\}$ if $r$ is a positive number such that $\{z:|z-\lambda|<2 r\} \cap \operatorname{sp}_{A}(x)=\{\lambda\}$. Then, by Cauchy's theorem,

$$
\begin{aligned}
0 & =\frac{1}{2 \pi i} \int_{|z-\lambda|=r} P_{z}(x(1-g)) d z=\frac{1}{2 \pi i} \int_{|z-\lambda|=r} R_{z}(x)(1-g) d z \\
& =(1-g) f_{-1}(x)=f_{-1}(x)(1-g) .
\end{aligned}
$$

We see that $f_{-1}(x)=f_{-1}(x) g=g f_{-1}(x)$. Then $f_{-p-1}(x)=(\lambda-x)^{p} f_{-1}(x)=0$. Hence $\lambda$ is a pole of $R_{z}(x)$ of order less than or equal to $p$.

Now let $m \leq p$ be the order of the pole of $R_{z}(x)$ at $\lambda$. By Lemma 2.4, $\left(1-f_{-1}(x)\right) A=(\lambda-x)^{m} A$ and $R\left[A(\lambda-x)^{m}\right]=f_{-1}(x) A$. Then since $f_{-1}(x)$ commutes with $x$ and $m \geq 1,(\lambda-x)$ generates an $\left(m, f_{-1}(x), R\right)$-decomposition of $A$, but $p$ is the smallest such integer so $p=m$. This proves (c). Then (b) follows from Lemma 2.4. Since

$$
\begin{aligned}
g\left(1-f_{-1}(x)\right) & =g(x-\lambda) f_{0}(x)=g(x-\lambda)^{p} f_{p-1}(x) \\
& =0 \cdot f_{p-1}(x)=0,
\end{aligned}
$$

we see that $f=f_{-1}(x) g=f_{-1}(x)$. This proves (d).

2.7. Theorem. Let $x$ be in A. For a positive integer $p$ and a complex number $\lambda$ these conditions are equivalent:

(a) $\lambda$ is a pole of $R_{x}(x)$ of order $p$. 
(b) There is a nonzero idempotent $g$ in $A$ such that $(\lambda-x)$ generates a $(p, g, R)$-decomposition of $A$, and $p$ is the smallest positive integer such that this is true for $x$ and $\lambda$.

(c) There is a nonzero idempotent $b$ in $A$ such that $(\lambda-x)$ generates a $(p, b, L)$-decomposition of $A$, and $p$ is the smallest positive integer sucb that this is true for $x$ and $\lambda$.

Proof. Conditions (a) and (b) are equivalent by Lemmas 2.4 and 2.5. By Lemma 2.4 we know that (a) implies (c). The proof that (c) implies (a) is similar to that given for Lemma 2.5 .

3. Riesz points of the spectrum. The singularities we are about to discuss are analogous to those considered by Lay in [7] and by West in [14]. They are especially easy to handle algebraically and will be important in the work taken up in $\$ 4$.

3.1. Definition. For $x$ in $A$, a number $\lambda$ in $\mathrm{sp}(x)$ is called a Riesz point of $\mathrm{sp}(x)$ if and only if $\lambda$ is a pole of $R_{z}(x)$ of finite rank.

3.2. Lemma. If $\lambda$ is in $\mathrm{sp}(x), \lambda$ is a Riesz point of $\mathrm{sp}(x)$ if and only if there is a positive integer $p$ and an idempotent $g \neq 0$ in $S_{A}$ sucb that $(\lambda-x)$ generates $a(p, g, R)$-decomposition of $A$ or, equivalently, $(\lambda-x)$ generates a $(p, g, L)$-decomposition of $A$. For sucb numbers $\lambda,(\lambda-x)$ is a Fredbolm element of index zero.

Proof. Suppose $\lambda$ is a Riesz point of $\mathrm{sp}(x)$. Let $p$ be the order of the pole of $R_{z}(x)$ at $\lambda$ and let $f$ denote the spectral idempotent for $x$ at $\lambda$. By Lemma 2.4, $R\left[A(\lambda-x)^{p}\right]=f A$ and $L\left[(\lambda-x)^{p} A\right]=A f$. Since $f$ is finite, $\theta\left(L\left[(\lambda-x)^{p} A\right]\right)=$ $\theta(f)=\theta\left(R\left[A(\lambda-x)^{p}\right]\right)<\infty$. Hence $\lambda-x$ is a Fredholm element of index zero. By Theorem 2.7, the element $\lambda-x$ generates a $(p, g, R)$-decomposition of $A$ and a $(p, b, L)$-decomposition for some nonzero idempotents $g$ and $b$ and, by the preceding remarks, $g$ and $b$ must be finite.

Conversely, if $\lambda-x$ generates a $(p, g, R)$-decomposition for some $p \geq 1$ and some finite idempotent $g$ then, by Theorem $2.7, \lambda$ is a pole of $R_{z}(x)$ of order $p$. Again let $f$ denote the spectral idempotent for $x$ at $\lambda$. By Lemma 2.4, $f A=$ $R\left[A(\lambda-x)^{p}\right]$ which is a right ideal of finite order. Hence $f$ is in $S_{A}$ and $\lambda$ is a Riesz point. If $\lambda-x$ generates a $(p, b, L)$-decomposition for some $p \geq 1$ and nonzero finite idempotent $b$, the proof that $\lambda$ is a Riesz point is similar.

3.3. Lemma. Let $N$ be a closed two-sided ideal of $A$ and let $\phi: A \rightarrow A / N$ be the quotient bomomorphism. For each $x$ in $A$, if $f(z)$ is a complex-valued function analytic on an open set containing $\operatorname{sp}(x)$ then $\phi(f(x))=f(\phi(x))$. 
Proof. It is sufficient to show $\operatorname{sp}_{A}(x) \subseteq \operatorname{sp}_{A / N}(x+N)$. Let $W$ be an open set containing $\operatorname{sp}_{A}(x)$ and let $\gamma$ be an admissible cycle for $(W, \operatorname{sp}(x))$. Then $\gamma$ is an admissible cycle for $\left(W, \operatorname{sp}_{A} / N(x+N)\right)$. If $z$ is not in $\operatorname{sp}_{A / N}(x+N)$ let $P_{z}(\phi(x))=P_{z}(x+N)=\phi(z-x)^{-1}$. Then, if $z \notin \operatorname{sp}_{A}(x), P_{z}(x+N)=\phi\left(R_{z}(x)\right)$. Compute

$$
\begin{aligned}
f(\phi(x)) & =\frac{1}{2 \pi i} \int_{\gamma} f(z) P_{z}(x+N) d z=\frac{1}{2 \pi i} \int_{\gamma} f(z)\left(\phi\left(R_{z}(x)\right) d z\right) \\
& =\phi\left(\frac{1}{2 \pi i} \int_{\gamma} f(z) R_{2}(x) d z\right)=\phi(f(x)),
\end{aligned}
$$

since the quotient mapping $\phi$ is continuous.

3.4. Lemma. If $\lambda$ is a Riesz point of $\mathrm{sp}(x)$ and $N$ is a closed two-sided ideal of $A$ such that $A / N$ is semisimple, either $\lambda-x$ is invertible modulo $N$ or $\lambda$ is a Riesz point in $\operatorname{sp}_{A / N}(x+N)$.

Proof. For $N$ such a closed ideal of $A, \phi: A \rightarrow A / N$ is the quotient homomorphism. If $\phi(\lambda-x)$ is not invertible $\lambda$ is an isolated point of $\operatorname{sp}_{A} / N(\phi(x)) \subseteq$ $\operatorname{sp}_{A}(x)$. For each integer $m$ construct the function $f_{m}(z)$ for $x$ at $\lambda$ as in $\$ 2.1$. Since $f_{-1}(x)$ is in $S_{A}, \phi\left(f_{-1}(x)\right)$ is in $S_{A ; N}$. By Lemma 3.3, $f_{m}(\phi(x))=\phi\left(f_{m}(x)\right)$ for all $m$, so $f_{-1}(\phi(x))$ is in $S_{A / N}$. If $p$ is the order of the pole of $R_{z}(x)$ at $\lambda$,

$$
\phi(0)=\phi\left(f_{-p-1}(x)\right)=f_{-p-1}(\phi(x)) .
$$

Let $P_{z}(\phi(x))=(\phi(z-x))^{-1}$ for all $z \notin \mathrm{sP}_{A} / N(\phi(x))$. Then $\lambda$ is a pole of $z \rightarrow$ $P_{z}(\phi(x))$ of order less than or equal to $p$ and rank less than or equal to $\theta\left(f_{-1}(x)\right)$. Hence $\lambda$ is a Riesz point of $\operatorname{sp}_{A / N}(\phi(x))$.

3.5. Theorem. Let $\lambda$ be a Riesz point of $\mathrm{sp}(x)$ and let $f$ be the spectral idempotent for $x$ at $\lambda$. Then $f$ is in $S_{A}$ and $\lambda-x-f$ is invertible in $A$.

Proof. Let $p$ be the order of the pole of $R_{z}(x)$ at $\lambda$. By definition of a Riesz point, $f$ is in $S_{A}$ and it is a nonzero element of $R\left[A(\lambda-x)^{p}\right] \cap L\left[(\lambda-x)^{p} A\right]$. Suppose $\lambda-x-f$ is not invertible. It is at least Fredholm (of index zero) by Lemma 3.2. There is a minimal idempotent $b$ in $A$ such that either

$$
b(\lambda-x-f)=0 \text { or }(\lambda-x-f) b=0,
$$

by [3, Theorem 2.3]. Assume $b(\lambda-x-f)=0$. Then $b(\lambda-x)=b f$. Therefore

$$
\begin{aligned}
0 & =b(\lambda-x)^{p} f=b f(\lambda-x)^{p-1} f=b(\lambda-x)^{p-1} f \\
& =b(\lambda-x)^{p-2} f=\cdots=b f .
\end{aligned}
$$

Then $0=b(\lambda-x)$ and so $b$ is in $L[(\lambda-x) A] \subseteq L\left[(\lambda-x)^{p} A\right]=A f$. Hence $b=b f$ $=0$, which is a contradiction. 
3.6. Definition. Let

$$
\begin{aligned}
& \Phi_{l}^{R}=\left\{x \in \Phi_{A}: a_{l}(x)=\delta_{l}(x)<\infty\right\}, \\
& \Phi_{r}^{R}=\left\{x \in \Phi_{A}: a_{r}(x)=\delta_{r}(x)<\infty\right\}, \\
& \Phi^{R}=\left\{x \in \Phi_{A}: a_{r}(x)=\delta_{r}(x)=a_{l}(x)=\delta_{l}(x)<\infty\right\} .
\end{aligned}
$$

Using the theory developed in this section we will soon show that these three sets are equal.

3.7. Theorem. If $x$ is an element of $A$ and $\lambda$ is any complex number such that $\lambda-x$ is in $\Phi_{l}^{R}$ or $\Phi_{r}^{R}$, then either $\lambda-x$ is invertible or $\lambda$ is a Riesz point of $\operatorname{sp}(x)$.

Proof. Assume $\lambda$ is in sp $(x)$ and that $\lambda-x$ is in $\Phi_{l}^{R}$. Let $n=a_{l}(\lambda-x)=$ $\delta_{l}(\lambda-x)<\infty$. Since $\lambda-x$ is a Fredholm element so is $(\lambda-x)^{n}$. Let $g$ and $b$ be idempotents in $S_{A}$ such that $A(1-g)=A(\lambda-x)^{n}$ and $(1-b) A=(\lambda-x)^{n} A$. Then

$$
R\left[A(\lambda-x)^{n}\right]=g A \text { and } L\left[(\lambda-x)^{n} A\right]=A b .
$$

By Corollary 2.3,

$$
g A \cap(\lambda-x)^{n} A=\{0\} \text { and } A=(\lambda-x)^{n} A \oplus g A .
$$

Hence

$$
g A \cap(1-b) A=\{0\} \text { and } A=(1-b) A \oplus g A \text {. }
$$

Each of the summands is closed because $g$ and $b$ are idempotents.

Since $A$ is a Banach algebra, there exist projections $P_{1}$ and $P_{2}$ in $B(A)$ such that

$$
P_{1}(g z+(1-b) y)=g z \text { and } P_{2}(g z+(1-b) y)=(1-b) y
$$

for all $z$ and $y$ in $A$. Since $(\lambda-x)^{n+1} A=(\lambda-x)^{n} A$ we see that $(\lambda-x)(1-b) A$ $=(1-b) A$. In particular, $(\lambda-x)(1-b)=(1-b)(\lambda-x)(1-b)$. Then $(1-b) A$ is invariant under $L_{(\lambda-x)}$, the operator of left mulitplication by $\lambda-x$ on $A$. Also

$$
\begin{aligned}
(\lambda-x) g A & =(\lambda-x) R\left[A(\lambda-x)^{n+1}\right]=\left\{(\lambda-x) y:(\lambda-x)^{n+1} y=0\right\} \\
& \subseteq R\left[A(\lambda-x)^{n}\right]=g A .
\end{aligned}
$$

So $g A$ is also invariant under $L_{(\lambda-x)^{\circ}}$. Then $P_{1} L_{(\lambda-x)}=L_{(\lambda-x)} P_{1}$ and $P_{2} L_{(\lambda-x)}$ $=L_{(\lambda-x)} P_{2}$. If there is a $y$ in $A$ such that $0=(\lambda-x)^{n}(1-b) y$, then $(1-b) y$ is in $R\left[A(\lambda-x)^{n}\right]=g A$. We have shown that $(1-b) A \cap g A=\{0\}$, so $(1-b) y$ must be 0 . Hence $L_{(\lambda-x)^{n}}:(1-b) A \rightarrow(1-b) A$ is a bijection. There is an operator $K$ in $B((1-b) A)$ such that 


$$
L_{(\lambda-x)^{n}} K((1-b) x)=(1-b) x=K L_{(\lambda-x)^{n}}((1-b) x)
$$

for all $x$ in $A$. Extend $K$ to be identically zero on $g A$. Then $L(\lambda-x)^{n} K P_{2}=$ $K L_{(\lambda-x) n} P_{2}=P_{2}$.

Let $B=\left\{T\right.$ in $B(A): P_{1} T=T P_{1}$ and $\left.P_{2} T=T P_{2}\right\}$, i.e. the subalgebra of operators having $(1-b) A$ and $g A$ as invariant subspaces. $B$ is a semisimple Banach algebra with unit I and with the inherited norm topology. Notice that $L_{(\lambda-x)}, L_{x}$, $K, P_{1}$, and $P_{2}$ are all in $B$.

Now clearly $P_{1} B$ is contained in $R_{B}\left[B\left(\lambda I-L_{x}\right)^{n}\right]$. If $T$ is in $R_{B}\left[B\left(\lambda I-L_{x}\right)^{n}\right]$ and if $P_{2} T$ is not zero there is a nonzero $z$ in $A$ such that $0 \neq P_{2} T(z)=$ $P_{2} T P_{2}(z)$. For some $y$ in $A, P_{2} T P_{2}(z)=(\lambda-x)^{n} y \neq 0$. But

$$
\begin{aligned}
0 & =\left(\lambda I-L_{x}\right)^{n} T P_{2}(z)=\left(\lambda I-L_{x}\right)^{n} P_{2} T P_{2}(z) \\
& =(\lambda-x)^{n}(\lambda-x)^{n} y=(\lambda-x)^{2 n} y .
\end{aligned}
$$

Then $0 \neq(\lambda-x)^{n} y \in R\left[A(\lambda-x)^{n}\right] \cap(\lambda-x)^{n} A=\{0\}$, a contradiction. Hence, for for all $T$ in $R_{B}\left[B\left(\lambda I-L_{x}\right)^{n}\right], 0=P_{2} T=\left(I-P_{1}\right) T$ so we see $R_{B}\left[B\left(\lambda I-L_{x}\right)^{n}\right]=$ $P_{1} B$. For any $T$ in $B$,

$$
T=P_{2} T+P_{1} T=L_{(\lambda-x)^{n}} K P_{2} T+P_{1} T,
$$

which is in $\left(\lambda I-L_{x}\right)^{n} B \oplus P_{1} B \subseteq P_{2} B \oplus P_{1} B$, so $B=P_{1} B \oplus\left(\lambda I-L_{x}\right)^{n} B$. Hence $\left(\lambda I-L_{x}\right)$ generates an $\left(n, P_{1}, R\right)$-decomposition of the algebra $B$. Let $p$ be the smallest positive integer such that $\left(\lambda I-L_{x}\right)$ generates a $(p, G, R)$-decomposition of $B$ for some nonzero idempotent $G$ in $B$. By Lemma $2.6, \lambda$ is a pole of $R_{z}\left(L_{x}\right)$ of order $p$ and $G$ is the spectral idempotent for $L_{x}$ at $\lambda$.

Now $\operatorname{sp}_{B}\left(L_{x}\right)=\operatorname{sp}_{B(A)}\left(L_{x}\right)=s \mathrm{p}(x)$ by [9, Theorem 1.6.9]. The number $\lambda$ is isolated in this set. For each integer $m$ construct the open set $U$ containing sp $(x)$ and functions $f_{m}(z)$ at $\lambda$ as in (2.1). Let $\gamma$ be an admissible cycle for $(U, \operatorname{sp}(x))$. For all $z$ not in $\mathrm{sp}(x)$ we know $R_{z}\left(L_{x}\right)=L_{R_{z}(x)}$. Then, for each integer $m$,

$$
\begin{aligned}
f_{m}\left(L_{x}\right) & =\frac{1}{2 \pi i} \int_{\gamma} f_{m}(z) R_{z}\left(L_{x}\right) d z=\frac{1}{2 \pi i} \int_{\gamma} f_{m}(z) L_{R_{z}(x)} d z \\
& =L_{\left((2 \pi i)^{-1} \int_{\gamma} f_{m}(z) R_{z}(x) d z\right)}=L_{f_{m}(x)}
\end{aligned}
$$

since the mapping $y \rightarrow L_{y}$ from $A$ into $B(A)$ is continuous.

Now $0 \neq f_{-p}\left(L_{x}\right)$ and $f_{-p-1}\left(L_{x}\right)=0$, so $0 \neq L_{f_{-p}(x)}$ and $L_{f_{-p-1}(x)}=0$. Then $f_{-p}(x) \neq 0$, and since $A$ is semisimple, $f_{-p-1}(x)=0$. Hence $\lambda$ is a pole of $R_{z}(x)$ of order $p$. By Theorem 2.7, $n=p$. So, $P_{1}=f_{-1}\left(L_{x}\right)=L_{f_{-1}(x)}$. Then 
for all $y$ in $A, P_{1}(y)=f_{-1}(x) y$. Hence $g A=f_{-1}(x) A$. Therefore $f_{-1}(x)$ is in $S_{A}$ and $\theta(g)=\theta\left(f_{-1}(x)\right)$. If $\lambda-x$ is a singular element of $\Phi_{r}^{R}$ the proof that $\lambda$ is a Riesz point of $\mathrm{sp}(x)$ is similar.

3.8. Corollary. $\Phi_{l}^{R}=\Phi^{R}=\Phi_{r}^{R}$.

Proof. Let $y$ be in $\Phi_{l}^{R}$. If $y$ is invertible, $0=a_{l}(y)=\delta_{l}(y)=a_{r}(y)=\delta_{r}(y)$ so $y$ is in $\Phi^{R}$. If $y$ is not invertible, 0 is a Riesz point of sp $(y)$ by Theorem 3.5. Let $n$ be the order of the pole of $R_{z}(y)$ at 0 . By Lemma 2.4, $n=a_{l}(y)=$ $\delta_{l}(y)=a_{r}(y)=\delta_{r}(y)$. Hence, $\Phi_{l}^{R} \subseteq \Phi^{R}$. Similarly, $\Phi_{r}^{R} \subseteq \Phi^{R}$. Clearly $\Phi^{R} \subseteq \Phi_{r}^{R} \cap$ $\Phi_{l}^{R}$.

3.9. Theorem. For $x$ in $A, c$ in $I$ the ideal of inessential elements of $A$, and $\lambda$ in $\operatorname{sp}(x)$ if $x c=c x$ and $\lambda-x-c$ is invertible, then $\lambda$ is a Riesz point of $\mathrm{sp}(x)$.

Proof. Let $k=-(\lambda-x-c)^{-1} c$. Since $x c=c x$ we have also $k=-c(\lambda-x-c)^{-1}$. Since $I$ is an ideal, $k$ is in $I$.

$$
1-k=(\lambda-x-c)^{-1}(\lambda-x)=(\lambda-x)(\lambda-x-c)^{-1} \text {. }
$$

For all integers $m \geq 0$,

$$
\begin{aligned}
A(1-k)^{m} & =A\left((\lambda-x-c)^{-1}(\lambda-x)\right)^{m} \\
& =A(\lambda-x-c)^{-m}(\lambda-x)^{m}=A(\lambda-x)^{m} .
\end{aligned}
$$

Similarly $(1-k)^{m} A=(\lambda-x)^{m} A$ for all integers $m \geq 0$. Using a theorem of Barnes [2, Theorem 3.3], $\infty>\alpha_{l}(1-k)=\delta_{l}(1-k)=\alpha_{r}(1-k)=\delta_{r}(1-k)$. Let this integer be $p$. Since $(\lambda-x)$ is not invertible, $p$ is greater or equal to one. By [2, Theorem 3.5], $1-k$ is a Fredholm element of index 0 . There are idempotents $e$ and $f$ in $s_{A}$ such that $\theta(f)=\theta(e)$,

$$
A(1-e)=A(1-k)=A(\lambda-x) \text { and }(1-f) A=(1-k) A=(\lambda-x) A .
$$

Then $\lambda-x$ is Fredholm of index 0 and $p=\alpha_{l}(\lambda-x)=\delta_{l}(\lambda-x)=\alpha_{r}(\lambda-x)=$ $\delta_{r}(\lambda-x)$. We see that $\lambda-x$ is in $\Phi^{R}$. By Theorem $3.7, \lambda$ is a Riesz point of $\operatorname{sp}(x)$.

The following corollary is immediate from Theorems 3.5 and 3.9.

3.10. Corollary. A complex number $\lambda$ is a Riesz point of $\mathrm{sp}(x)$ if and only if there is an element $c$ in $I_{A}$ such that $x c=c x$ and $\lambda-x-c$ is invertible in $A$.

3.11. Corollary. A complex number $\lambda$ is a Riesz point of $\mathrm{sp}(x)$ if and only if $\lambda-x$ is a singular element of $\Phi^{R}$.

Proof. If $\lambda-x$ is a singular element of $\Phi^{R}, \lambda$ is a Riesz point of $\operatorname{sp}(x)$ by Theorem 3.7. Suppose $\lambda$ is a Riesz point in $\operatorname{sp}(x)$. Then, by Lemmas 3.2 and 
2.6, $\lambda-x$ is a Fredholm element and $\alpha_{l}(\lambda-x)=\delta_{l}(\lambda-x)<\infty$. Then $\lambda-x$ is in $\Phi^{R}$.

4. Riesz elements of $A$.

4.1. Definition. An element $y$ of a normed algebra is called a topological nilpotent or a quasi-nilpotent if and only if

$$
\lim _{n \rightarrow \infty}\left\|y^{n}\right\|^{1 / n}=0 \text {. }
$$

If $A$ is a semisimple Banach algebra, $S_{A}$ its socle, and $\pi: A \rightarrow A / \bar{S}_{A}$ the quotient homomorphism, an element $y$ of $A$ is called a Riesz element of $A$ if and only if $\pi(y)$ is a topological nilpotent. Let $R_{A}$ denote the Riesz elements of $A$.

T. T. West has shown $[14, \S \S 5$ and 6$]$ that $R_{A}$ is not closed under the operations of addition and multiplication if $A$ is $B(X)$ for some infinite-dimensional Banach space $X$, nor is $R_{B(X)}$ closed in the uniform topology; but if $H$ is an infinite-dimensional Hilbert space, $B(H)$ is the algebra generated by $\Re_{B(H)}$.

For each $y$ in $A$ let $\mathcal{F}_{y}=\left\{\lambda \in \mathrm{C}: \lambda-y \in \Phi_{A}\right\}$. $\mathcal{F}_{y}$ is called the generalized Fredholm region for $y$. When $A$ is infinite-dimensional, as we are always assuming it to be, W. Pfaffenberger [8, Chapter 3] has shown $y$ is in $R_{A}$ if and only if $\mathcal{F}_{y}$ is the set of nonzero complex numbers. He also showed that $I_{A}$ is contained in $R_{A}[8]$.

Several theorems which follow in this section are due to B. Barnes. Some of them have not been published in this form although they are similar to theorems given in [2].

4.2. Lemma (Barnes [3, Proposition 2.2]). If $u$ is in the closure of $S_{A}$ then $1-u$ is invertible modulo $S_{A}$.

4.3. Lemma (Barnes). If $x$ is a Riesz element of $A$ then $1-x$ is invertible $\operatorname{modulo} S_{A}$.

Proof. Since $X$ is a Riesz element of $A, \pi(x)$ is a topological nilpotent in the Banach algebra $\pi(A)=A / \bar{S}_{A}$. For all $\lambda \neq 0, \pi(x) / \lambda$ is quasi-regular. Let $v$ be in $A$ such that

$$
\pi(1-x) \pi(1-v)=\pi(1)=\pi(1-v) \pi(1-x)
$$

There exist $s_{1}$ and $s_{2}$ in $\bar{S}_{A}$ such that

$$
(1-v)(1-x)=\left(1-s_{1}\right) \text { and }(1-x)(1-v)=\left(1-s_{2}\right) \text {. }
$$

By Lemma 4.2 there exist $c_{1}$ and $c_{2}$ in $S_{A}$ and $y_{1}$ and $y_{2}$ in $A$ such that $\left(1-y_{1}\right)\left(1-s_{1}\right)=\left(1-c_{1}\right)$ and $\left(1-s_{2}\right)\left(1-y_{2}\right)=\left(1-c_{2}\right)$. Therefore

$$
\left(1-y_{1}\right)(1-v)(1-x)=\left(1-c_{1}\right) \text { and }(1-x)(1-v)\left(1-y_{2}\right)=\left(1-c_{2}\right) \text {. }
$$


The following lemma was proved by Taylor in much greater generality than is is needed here, and using the notation of operator theory. The proof given below is merely a translation into algebra notation.

4.4. Lemma (Taylor [13]). $a_{l}(x)<\infty$ if and only if $R[A x] \cap x^{n} A=\{0\}$ for some $n \geq 1$; and $a_{r}(x)<\infty$ if and only if $L[x A] \cap A x^{n}=\{0\}$ for some $n \geq 1$.

Proof. If $\alpha_{l}(x)=\infty$, then $R[A x] \neq R\left[A x^{n+1}\right]$ for all $n \geq 1$. So, for each $n \geq 1$, we may choose $0 \neq y_{n}$ in $R\left[A x^{n+1}\right]$ which is not in $R\left[A x^{n}\right]$. Let $z_{n}=x^{n} y_{n} \neq 0$ for all $n \geq 1$. Then $z_{n}$ is in $R[A x] \cap x^{n} A$ for all $n \geq 1$.

Conversely if $R[A x] \cap x^{n} A \neq\{0\}$ for all $n \geq 1$, then for all $n$ there is a $y_{n}$ in $A$ such that $x^{n} y_{n} \neq 0$ and $x^{n+1} y=0$. Then $y_{n}$ is in $R\left[A x^{n+1}\right]$ but not in $R\left[A x^{n}\right]$. Hence $a_{l}(x)=\infty$. The second statement is proved similarly.

4.5. Proposition (Barnes). Let $x$ be a Riesz element of A. Then $a_{l}(\lambda-x)$ and $\alpha_{r}(\lambda-x)$ are finite for all nonzero $\lambda$.

Proof. It is sufficient to show $a_{l}(1-x)$ and $a_{r}(1-x)$ are finite. We prove $a_{l}(1-x)<\infty$. The proof that $a_{r}(1-x)<\infty$ is similar.

For each positive integer $n$ let $K_{n}=R[A(1-x)] \cap(1-x)^{n} A$. If $\alpha_{l}(1-x)$ is not finite then, by Lemma $4.4, K_{n} \neq\{0\}$ for all $n \geq 1$. Since $(1-x)$ is in $\Phi(A)$ by Lemma 4.3, $R[A(1-x)]$ is of finite order. We have $K_{n+1} \subseteq K_{n} \subseteq R[A(1-x)]$ for all positive $n$. By $[2, \mathrm{p} .498]$ there is a $p \geq 1$ such that $K_{n}=K_{p}$ for all $n \geq p$. By assumption $\{0\} \neq K_{p}$, let $e$ be a minimal idempotent of $A$ contained in $K_{p}$. For all $n \geq p$,

$$
e \in R[A(1-x)] \cap A e \cap(1-x)^{n} A \subseteq K_{n}=K_{p} \subseteq K_{p-1} \subseteq K_{1} .
$$

Then, for all positive integers $n, e$ is in $K_{n} e$.

For each $v$ in $A$ define an operator $T_{\nu}: A e \rightarrow A e$ by $T_{v}(x e)=v x e$ for all $x$ in $A$. Then $T_{v}$ is in $B(A e)$ for all $v$ in $A$; and clearly $\left|T_{v}\right| \leq\|v\|$ for all $v$ in $A$, where $1 . \mid$ denotes the operator norm in $B(A e)$.

Choose a sequence $\left\{s_{m}\right\}_{m=1}^{\infty}$ in $s_{A}$ such that $\lim _{m \rightarrow \infty}\left\|x^{m}-s_{m}\right\|^{1 / m}=0$. Then, for each $m, T_{s_{m}}$ is an operator with finite-dimensional range in $B(A e)$ and

$$
\left|\left(T_{x}\right)^{m}-T_{s_{m}}\right|^{1 / m}=\left|T_{\left(x^{m}-s_{m}\right)}\right|^{1 / m} \leq\left\|x^{m}-s_{m}\right\|^{1 / m} .
$$

Hence $T_{x}$ is a Riesz operator in $B(A e)$. The mapping $T_{1}$ is the identity operator on Ae. By [10, Lemma 3.2, p. 323] the ascent of $T_{1}-T_{x}$ is finite. Then there is an integer $q$ such that the nullity of $T_{1}-T_{x}$ and the range of $\left(T_{1}-T_{x}\right)^{q}$ have only 0 in common; see $[13$, p. 22] or Lemma 3.4 . Then 


$$
\begin{aligned}
\{0\} & =\left\{y e \in A e:\left(T_{1}-T_{x}\right) y e=0\right\} \cap\left(T_{1}-T_{x}\right)^{q} A e \\
& =\{y e \in A e:(1-x) y e=0\} \cap(1-x)^{q} A e \\
& =R[A(1-x)] \cap A e \cap(1-x)^{q} A e=K_{q} e
\end{aligned}
$$

contradicting the fact that $0 \neq e \in K_{q} e$.

4.6. Lemma. Let $x$ be a Fredbolm element of $A$ such that $\alpha_{l}(x)=m<\infty\left(a_{p}(x)\right.$ $=n<\infty)$ then $\delta_{r}(x)=m\left(\delta_{l}(x)=n\right)$.

Proof. Since $x$ is Fredholm, $x^{m}$ is in $\Phi(A)$ where $\alpha_{l}(x)=m$. There is an idempotent $e_{m}$ in $S_{A}$ such that $R\left[A x^{m}\right]=e_{m} A$. For all integers $n \geq m, x^{n}-e_{m}$ is in $\Phi(A)$. By [3, Theorem 2.3] if $x^{n}-e_{m}$ is not left invertible in $A$ there is a minimal idempotent $g_{n}$ in $A$ such that $\left(x^{n}-e_{m}\right) g_{n}=0$. In this case $x^{m+n} g_{n}=$ $x^{m} e_{m} g_{n}=0 g_{n}=0$. Then $g_{n} \in R\left[A\left(x^{m+n}\right)\right]=R\left[A x^{m}\right]=e_{m} A$, so that $g_{n}=e_{m} g_{n}=$ $x^{n} g_{n}=0$, contradicting the fact that $g_{n}$ is a minimal idempotent. Hence $x^{n}-e_{m}$ is left invertible in $A$ for all $n \geq m$.

Now fix $n \geq m$. For each $y$ in $A$ there is a $z_{y}$ in $A$ such that $y=z_{y}\left(x^{n}-e_{m}\right)$. If $y$ is in $A x^{m}, 0-y e_{m}=z_{y}\left(x^{n}-e_{m}\right) e_{m}=-z_{y} e_{m} \cdot$ Then $y=z x^{n} \in A x^{n}$. This shows that $\delta_{r}(x)$ is less than or equal to $m$. Let $k=\delta_{r}(x)$. Ax $=A x^{m}$ so we have

$$
R\left[A x^{k+1}\right]=R\left[A x^{k}\right]=R\left[A x^{m}\right] \text {. }
$$

Then $k=m$ by definition of $a_{l}(x)$.

4.7. Theorem. Let $x$ be a Riesz element of A. Then every nonzero point of $\mathrm{sp}(x)$ is a Riesz point.

Proof. Suppose $\lambda \neq 0$ is in $\operatorname{sp}(x)$. Then $x / \lambda$ is a Riesz element of $A$. Then, us ing Lemma 4.3 and Proposition 4.5, we see that $(1-x / \lambda)$ is in $\Phi(A)$, so $\lambda-x$ is also, and $\alpha_{l}(\lambda-x)$ and $\alpha_{r}(\lambda-x)$ are finite. Then, by Lemma 4.6, $a_{l}(\lambda-x)=$ $\delta_{r}(\lambda-x)<\infty$ and $\alpha_{r}(\lambda-x)=\delta_{l}(\lambda-x)<\infty$ similarly. Then, by Corollary 2.3,

$$
\alpha_{l}(\lambda-x)=\delta_{l}(\lambda-x)=\alpha_{r}(\lambda-x)=\delta_{r}(\lambda-x)<\infty .
$$

So we have $(\lambda-x)$ in $\Phi^{R}$. Using Theorem 3.7 we see that $\lambda$ is a Riesz point of $\operatorname{sp}(x)$.

4.8. Corollary (Barnes). If $x$ is a Riesz element of $A$, the spectrum of $x$ is finite or a sequence converging to zero.

4.9. Corollary (Barnes). If $x$ is a Riesz element of $A, 1-x$ is Fredbolm of index 0 . 
Proof. If $1-x$ is invertible then it is certainly Fredholm of index 0 . If 1 is in $\operatorname{sp}(x)$ it is a Riesz point; so, by Corollary $3.11,1-x$ is Fredholm of index 0 .

We will show in this section that $x$ is in $R_{A}$ if and only if every nonzero point of $\mathrm{sp}(x)$ is a Riesz point. This was shown by $A$. Ruston in the case that $B(X)=A$ for some Banach space $X$; see $[14, \mathrm{p} .133]$. We will need the following lemma.

4.10. Lemma. Let $\lambda_{1}$ and $\lambda_{2}$ be two distinct Riesz points in sp $(x)$. Let $g_{1}$ and $g_{2}$ be the respective spectral idempotents for $x$ at $\lambda_{1}$ and $\lambda_{2}$. Then $g_{1} g_{2}=$ $0=g_{1} g_{2}$.

Proof. $\lambda_{1}$ and $\lambda_{2}$ are distinct isolated points of $\mathrm{sp}(x)$. There exist positive numbers $r_{1}$ and $r_{2}$ such that

$$
\left\{\lambda_{1}, \lambda_{2}\right\}=\operatorname{sp}(x) \cap \bigcup_{i=1}^{2}\left\{z:\left|z-\lambda_{i}\right|<2 r_{i}\right\} .
$$

Let

$$
\begin{aligned}
W_{i} & =\left\{z:\left|z-\lambda_{i}\right|<r_{i}\right\} \quad(i=1,2) \\
V & =\left\{z:\left|z-\lambda_{1}\right|>r_{1} \text { and }\left|z-\lambda_{2}\right|>r_{2}\right\} .
\end{aligned}
$$

Let $U=V \cup W_{1} \cup W_{2}$. Then $\operatorname{sp}(x) \subseteq U$. If $z$ is in $U$, let

$$
\chi_{i}(z)= \begin{cases}1 & \text { if } z \text { is in } W_{i}(i=1,2), \\ 0 & \text { otherwise. }\end{cases}
$$

Then $\chi_{1}$ and $\chi_{2}$ are analytic on $U$. Let $\gamma$ be an admissible cycle for $(U, \operatorname{sp}(x))$. Then

$$
g_{j}=\chi_{j}(x)=\frac{1}{2 \pi i} \int_{\gamma} \chi_{j}(z) R_{z}(x) d z \quad(j=1,2)
$$

Hence $g_{1} g_{2}=\chi_{1}(x) \chi_{2}(x)=\left(\chi_{1} \chi_{2}\right)(x)=0(x)=0$.

Let $x$ be an element of $A$ such that every nonzero point in $\operatorname{sp}(x)$ is a Riesz point. Then for any positive $\epsilon$ let $p_{\epsilon}=\{z:|z| \geq \epsilon\} \cap \operatorname{sp}(x) . p_{\epsilon}$ is either empty or finite, since Riesz points are isolated. Either $p_{\epsilon}$ is empty for some $\epsilon>0$ or else $\boldsymbol{x}$ is a topological nilpotent in $A$, in which case $\boldsymbol{x}$ is certainly a topological nilpotent modulo $\bar{S}_{A}$, and hence is a Riesz element.

Suppose that, for some positive $\epsilon, p_{\epsilon}$ is nonempty. Let $\left\{\lambda_{1}, \lambda_{2}, \cdots, \lambda_{m}\right\}=$ $p_{\epsilon}$, for some integer $m \geq 1$. Assume that $\lambda_{i} \neq \lambda_{j}$ if $i \neq j$ and $1 \leq i, j \leq m$. For each $1 \leq j \leq m$, let $g_{j}$ be the spectral idempotent for $x$ at $\lambda_{j}$, and let $n_{j}$ be the order of the pole of $R_{z}(x)$ at $\lambda_{j}$. Let $t_{\epsilon}=x\left(1-\sum_{j=1}^{m} g_{j}\right)$. 
4.11. Lemma. $x, \epsilon, p_{\epsilon}$ and $t_{\epsilon}$ be as above. Then $\operatorname{sp}\left(t_{\epsilon}\right) \subseteq\{z:|z| \leq \epsilon\}$ and bence $\lim _{n \rightarrow \infty}\left\|t_{\epsilon}^{n}\right\| \leq \epsilon$.

Proof. By Lemma 4.10, $g_{i} g_{j}=0$ if $1 \leq i, j \leq m$ and $i \neq j$. Then $\Pi_{i=1}^{m}\left(1-g_{i}\right)$ $=1-\sum_{i=1}^{m} g_{i}$; and, for all $1 \leq i \leq m, A=g_{i} A \oplus\left(1-g_{i}\right) A$. Using induction on $m$ we see

$$
A=g_{1} A \oplus g_{2} A \oplus \cdots \oplus g_{m} A \oplus \prod_{i=1}^{m}\left(1-g_{i}\right) A .
$$

We want to show that, for each $|\mu| \geq \epsilon,\left(\mu-t_{\epsilon}\right)$ is invertible in $A$. By [9, (1.6.9)], it is enough to show that $R\left[A\left(\mu-t_{\epsilon}\right)\right]=\{0\}$ and $\left(\mu-t_{\epsilon}\right) A=A$.

Using the orthogonality of the idempotents $\left\{g_{i}\right\}_{i=1}^{m}$, any element $w$ of $A$ can be written in the form $w=\prod_{i=1}^{m}\left(1-g_{i}\right) w+\sum_{i=1}^{m} g_{i} w$. Compute using this fact and the definition of $t_{\epsilon}$ to see that

$$
\mu-t_{\epsilon}=\mu \sum_{i=1}^{m} g_{i}+(\mu+x) \prod_{i=1}^{m}\left(1-g_{i}\right) .
$$

Now for any $\mu \neq 0$ and for each $y$ in $R\left[A\left(\mu-t_{\epsilon}\right)\right]$ we have

$$
\begin{aligned}
0 & =\left(\mu-t_{\epsilon}\right) y \\
& =\left(\mu \sum_{i=1}^{m} g_{i}+(\mu-x)\left(1-\sum_{i=1}^{m} g_{i}\right)\right)\left(\sum_{i=1}^{m} g_{i} y+\left(1-\sum_{i=1}^{m} g_{i}\right) y\right) \\
& =(\mu-x)\left(1-\sum_{i=1}^{m} g_{i}\right) y+\mu \sum_{i=1}^{m} g_{i} y .
\end{aligned}
$$

This implies $\sum_{i=1}^{m} g_{i} y$ is in $\prod_{i=1}^{m}\left(1-g_{i}\right) A$. Then $\sum_{i=1}^{m} g_{i} y=0, y$ is in $\Pi_{i=1}^{m}\left(1-g_{i}\right) A$, and $0=(\mu-x) \Pi_{i=1}^{m}\left(1-g_{i}\right) y=(\mu-x) y$. Hence $R[A(\mu-t)] \subseteq$ $R[A(\mu-x)]$.

Now if $|\mu| \geq \epsilon$ there are two possible cases: either $\mu-x$ is invertible or $\mu$ is in $p_{\epsilon}$. The lemma will be proved if it is shown that in either case $\mu-t_{\epsilon}$ is invertible. Assume $\mu-x$ is invertible and $\mu \neq 0$. Then $\{0\}=R[A(\mu-x)]=$ $R\left[A\left(\mu-t_{\epsilon}\right)\right]$. For all $w$ in $A$ use (3) to see that

$$
w=\left(\mu-t_{\epsilon}\right)\left[\sum_{i=1}^{m} g_{i} \frac{w}{u}+\prod_{i=1}^{m}\left(1-g_{i}\right)(\mu-x)^{-1} w\right] .
$$

Hence $A=\left(\mu-t_{\epsilon}\right) A$. This shows that $\mu-t_{\epsilon}$ is invertible if $\mu-x$ is invertible.

If $\mu$ is in $p_{\epsilon}$, without loss of generality assume $\mu=\lambda_{1}$. Then

$$
g_{1} A=R\left[A\left(\lambda_{1}-x\right)^{n} 1\right] \supseteq R\left[A\left(\lambda_{1}-x\right)\right] \supseteq R\left[A\left(\lambda_{1}-t_{\epsilon}\right)\right]
$$


We have already proved that $\sum_{i=1}^{m} g_{i} R\left[A\left(\lambda_{1}-t\right)\right]=\{0\}$. Since $\left\{g_{1}, g_{2}, \cdots, g_{m}\right\}$ is a pairwise orthogonal set of idempotents this implies $\{0\}=R\left[A\left(\lambda_{1}-t_{\epsilon}\right)\right]$. It remains only to show $A=\left(\lambda_{1}-t_{\epsilon}\right) A$. Since $\left(\lambda_{1}-x\right)\left(1-g_{1}\right)$ is invertible in $\left(1-g_{1}\right) A\left(1-g_{1}\right)$ as in the proof of Theorem 2.6, there is a $c$ in $A$ such that

$$
\left(\lambda_{1}-x\right)\left(1-g_{1}\right) c\left(1-g_{1}\right)=1-g_{1}=\left(1-g_{1}\right) c\left(1-g_{1}\right)\left(\lambda_{1}-x\right) \text {. }
$$

Since $\lambda_{1}-t_{\epsilon}=\lambda_{1} \sum_{i=1}^{m} g_{i}+\left(\lambda_{1}-x\right)\left(1-\sum_{i=1}^{m} g_{i}\right)$ we see that, for all $w$ in $A$,

$$
\left(\lambda_{1}-t_{\epsilon}\right)\left[\sum_{i=1}^{m} g_{i} \frac{w}{\lambda_{1}}+\prod_{i=1}^{m}\left(1-g_{i}\right) c\left(1-g_{1}\right) w\right]=w .
$$

Hence $A=\left(\lambda_{1}-t_{\epsilon}\right) A$.

4.12. Theorem. Let $x$ be in $A$ such that every nonzero point of $\mathrm{sp}(x)$ is a Riesz point. Then $x$ is a Riesz element of $A$.

Proof. We have already noticed that if $p_{\epsilon}$ is empty for all positive $\epsilon$, then $x$ is a Riesz element. Now assume $p_{\epsilon}$ is nonempty for some $\epsilon>0$. Let $\left\{\lambda_{1}, \lambda_{2}, \ldots\right.$, $\left.\lambda_{m}\right\}$ be the distinct elements of $p_{\epsilon}$ and for each $1 \leq i \leq m$ let $g_{i}$ be the spectral idempotent for $x$ at $\lambda_{i}$ as in Lemma 4.11. Since $g_{j}$ is in $S_{A}$ for all $1 \leq j \leq m$, we see that, for all positive $\epsilon, \pi(x)=\pi(t)$. By Lemma 4.11,

$$
\lim _{n \rightarrow \infty}\left\|\pi(x)^{n}\right\|^{1 / n}=\lim _{n}\left\|\pi\left(t_{\epsilon}\right)^{n}\right\|^{1 / n} \leq \lim _{n}\left\|t_{\epsilon}^{n}\right\|^{1 / n} \leq \epsilon .
$$

Hence $\lim _{n \rightarrow \infty}\left\|\pi(x)^{n}\right\|^{1 / n}=0$.

4.13. Corollary. If $x$ is in $A$ these are equivalent conditions:

(a) $x$ is in $R_{A}$.

(b) Every nonzero point in $\mathrm{sp}(x)$ is a Riesz point. for $x$.

(c) Every nonzero complex number is in $\mathfrak{F}_{x}$, the generalized Fredbolm region

(d) For each nonzero complex number, $\lambda-x$ is in $\Phi^{R}$.

Proof. (a) $\Rightarrow$ (b) Theorem 4.7. (b) $\Rightarrow$ (a) Theorem 4.12. (a) $\Leftrightarrow$ (c) $[8$, Chapter 3]. (d) $\Leftrightarrow$ (b) Corollary 3.11 .

If $x$ is in $A$ and $p(x)$ is a polynomial with complex coef ficients and zero constant term it is possible to have $p(x)$ in $R_{A}$ without having $x$ in $R_{A}$. For example, let $x$ be the identity element and $p(z)=z-z^{2}$. Then $p(x)=0 \in R_{A}$ but 1 is not in $R_{A}$. (We are assuming $A$ is infinite-dimensional.)

4.14. Theorem. If $x$ is in $A$ and $f$ is a complex valued analytic on a neigh. borbood of $\operatorname{sp}(x)$ which bas no zeroes in the set $\operatorname{sp}(x) \sim\{0\}$ and if $f(x) \in R_{A}$ then $x$ is in $\Re_{A}$. 
Proof. Let $\pi: A \rightarrow A / \bar{S}_{A}$ be the quotient homomorphism. Then $\operatorname{sp}_{\pi(A)}(\pi(x))$ $\subseteq \operatorname{sp}_{A}(x)$ and $\{0\}=\operatorname{sp}_{\pi(A)}(\pi f(x))=\operatorname{sp}_{\pi(A)}(f(\pi(x)))$ by Lemma 3.3. By the spectral mapping theorem

$$
f\left(\operatorname{sp}_{\pi(A)}(\pi(x))\right)=\operatorname{sp}_{\pi(A)}(f(\pi(x))) .
$$

Then $\operatorname{sp}_{\pi(A)}(\pi(x))=\{0\}$. Hence $x$ is in $R_{A}$.

Lastly, here are descriptions of Riesz elements in the Banach algebras considered earlier in $\$ 1$.

4.15. Example. Let $A=B(X)$ for some Banach space $X$, let $K(X)$ be the ideal of compact operators and let $\mathcal{F}(X)$ be the ideal of operators with finite-dimensional range. A. F. Ruston [10] has shown that an operator is topologically nilpotent modulo $K(X)$ if and only if it is topologically nilpotent modulo $\overline{\mathcal{F}(X)}$. Since $S_{B(X)}=\mathcal{F}(X)$, an operator $T$ is topologically nilpotent modulo $K(X)$ if and only if it is a Riesz element of $B(X)$ [14].

4.16. Example. Let $\Delta$ be a compact Hausdorff space and let $X$ be a Banach space. Let $A$ be the algebra of continuous functions from $\Delta$ into $B(X)$ defined in Example 1.7. Let $b$ be in $A$. Clearly sp $(b)=\bigcup_{y \in \Delta} s p(b(y))$. Fix $\lambda$ in $s p(b)$. Suppose there is a finite set of isolated points $F_{\lambda}=\left\{y_{1}, y_{2}, \cdots, y_{n}\right\} \subseteq \Delta$ such that, for all $1 \leq i \leq n, \lambda$ is a Riesz point in $\operatorname{sp}\left(b\left(y_{i}\right)\right)$, and if $y_{0} \notin\left\{y_{1}, y_{2}, \cdots\right.$, $\left.y_{n}\right\}, \lambda \notin \mathrm{sp}\left(b\left(y_{0}\right)\right)$. Then $\lambda$ is an isolated point in sp $(b)$. Let $r$ be a positive number such that $\operatorname{sp}(b) \cap\{z:|z-\lambda| \leq 2 r\}=\{\lambda\}$. Then for all $1 \leq i \leq n$ the spectral idempotent for $b\left(y_{i}\right)$ at $\lambda$ is

$$
f_{i}=\frac{1}{2 \pi i} \int_{|z-\lambda|=r} R_{z}\left(b\left(y_{i}\right)\right) d z
$$

a nonzero idempotent in $\mathcal{F}(X)$. The spectral projection for $b$ at $\lambda$ is $f=$ $(2 \pi i)^{-1} \int|z-\lambda| \Rightarrow R_{z}(b) d z$. Since $A$ is an algebra of continuous functions, and $R_{z}(b)(y)=R_{z}(b(y))$ for all $z$ not in $\operatorname{sp}(b)$ and all $y$ in $\Lambda$,

$$
f(y)= \begin{cases}0 & \text { if } y \notin F, \\ f_{i} & \text { if } y=y_{i} \text { for some } 1 \leq i \leq n .\end{cases}
$$

Hence $f$ is in the socle of $A$. By Theorem 3.5, $(\lambda-b-f)(y)=\lambda I-b(y)-f(y)$ is invertible in $B(X)$ for all $y$ in $\Delta$. Let $U$ be the set of invertible operators in $B(X)$. Let $\phi: U \rightarrow U$ be the homeomorphism $\phi(T)=T^{-1}$. Let $g: \Delta \rightarrow B(X)$ be the mapping $g(y)=(\lambda I-b(y)-f(y))^{-1}$ for all $y$ in $\Delta$. Then $g$ is the composition of the two continuous mappings $\phi$ and $(\lambda-b-f)$. Hence $g$ is in $A$. Therefore $\lambda-b-f$ is invertible and, by Theorem 3.9, $\lambda$ is a Riesz point in $\operatorname{sp}(b)$.

Now suppose that $b$ is in $A$ and $\lambda$ is a Riesz point in sp $(b)$. Let $f$ be the spectral idempotent for $b$ at $\lambda$. Let $r$ be a positive number such that 


$$
\{\lambda\}=\operatorname{sp}(b) \cap\{z:|z-\lambda| \leq 2 r\} .
$$

Then $f=(2 \pi i)^{-1} \int|z-\lambda|=r R_{z}(b) d z$. By Lemma 3.4, $\lambda-b-f$ is invertible in $A$. Since $f$ is in $S_{A}$, there is an orthogonal set of minimal idempotents of $A,\left\{e_{1}, e_{2}\right.$, $\left.\cdots, e_{n}\right\}$, such that $f=\sum_{i=1}^{n} e_{i}$. As in Example 1.7, there is a finite set of isolated points $\left\{w_{1}, w_{2}, \cdots, w_{n}\right\} \subseteq \triangle$ and a finite set of projections with one-dimensional range $\left\{E_{1}, E_{2}, \cdots, E_{n}\right\} \subseteq B(X)$ such that if $i \neq j$ but $w_{i}=w_{j}$, then $E_{i} \neq E_{j}$, and, for all $1 \leq i \leq n$ and all $y$ in $\Delta$,

$$
e_{i}(y)= \begin{cases}0, & y \neq w_{i}, \\ E_{i}, & y=w_{i} .\end{cases}
$$

(Notice that we cannot assume the sets $\left\{w_{i}\right\}_{i=1}^{n}$ or $\left\{E_{i}\right\}_{i=1}^{n}$ contain distinct elements.) Now for each $y$ in $\Delta \sim\left\{w_{i}\right\}_{i=1}^{n}, f(y)=0$ and $\lambda I-b(y)=(\lambda-b-f)(y)$ is invertible in $B(X)$ so $\lambda$ is not in sp $(b(y))$. For $1 \leq i \leq n, f\left(w_{i}\right) \neq 0$, so $\lambda$ is in $\operatorname{sp}\left(b\left(w_{i}\right)\right) \subset \operatorname{sp}(b)$. Then $\lambda$ is an isolated point of $\operatorname{sp}\left(b\left(w_{i}\right)\right)$ for all $1 \leq i \leq n$. As before, $f\left(w_{i}\right)$ is the spectral idempotent for $b\left(w_{i}\right)$ at $\lambda$ for all $1 \leq i \leq n$. Now $\lambda I-b\left(w_{i}\right)-f\left(w_{i}\right)$ is invertible and $f\left(w_{i}\right) \in \mathcal{F}(X)$ so, by (3.10), $\lambda$ is a Riesz point in $\operatorname{sp}\left(b\left(w_{i}\right)\right)$ for all $1 \leq i \leq n$.

We see that for $b$ in $A$ and $\lambda$ in sp $(b), \lambda$ is a Riesz point if and only if there is a finite set of isolated points $F_{\lambda}$ contained in $\Delta$ such that $\lambda$ is a Riesz point in $\operatorname{sp}(b(y))$ for all $y$ in $F_{\lambda}$, and if $y$ is not in $F_{\lambda}$, then $\lambda$ is not in sp $(b(y))$. Clearly, the order of the pole of the resolvent mapping $z \rightarrow R_{z}(b)$ at $\lambda$ is the maximum of the orders of the poles of $z \rightarrow R_{z}(b(y))$ at $\lambda$ for all $y$ in $F_{\lambda}$.

Therefore an element $b$ of $A$ is a Riesz element of $A$ if and only if $b(y)$ is a Riesz operator for all $y$ in $\Delta$ and for each $0 \neq \lambda \in \mathrm{sp}(b)$ there is a finite set $F_{\lambda}$ of isolated points of $\Delta$ such that $\lambda \notin \mathrm{sp}(b(y))$ for all $y \notin F_{\lambda}$.

4.17. Example. Let $A$ be the algebra defined in Example 1.8. Let $T$ be an an operator in $A$. Suppose $\lambda$ is an isolated point in $\operatorname{sp}_{A}(T)=\operatorname{sp}_{B(X)}(T)$. Let $f$ be the spectral idempotent for $T$ at $\lambda$. Since $R_{z}(T)$ is in $A$ for all $z \notin \operatorname{sp}(T)$, $f$ is also in $A$. Since $S_{A}=A \rightarrow \mathcal{F}(X)$, we see that $f$ is in $S_{A}$ if and only if $f$ is in $\mathcal{F}(X)$. Then $\lambda I-T-f$ is invertible in $A$ if and only if it is invertible in $B(X)$. Hence, by Theorems 3.9 and 3.5, $\lambda$ is a Riesz point in $\operatorname{sp}(T)$ with respect to the algebra $A$ if and only if it is with respect to $B(X)$. Therefore $\mathscr{R}_{A}=\Re_{B(X)} \cap A$.

\section{BIBLIOGRAPHY}

1. B. A. Barnes, Modular annihilator algebras, Canad. J. Math. 18 (1966), 566-578. MR 33 \#2681.

2. - A generalized Fredholm theory for certain maps in the regular representations of an algebra, Canad. J. Math. 20 (1968), 495-504. MR 38 \#534.

3. - The Fredholm elements of a ring, Canad. J. Math. 21 (1969), 84-95. MR 38 \#5823. 
4. F. E. Browder, On the spectral the ory of elliptic differential operators. I, Math. Ann. $142(1960 / 61), 22-130$. MR 35 \#804.

5. N. Dunford and J. T. Schwartz, Linear operators. I. General theory, Pure and Appl. Math., vol. 7, Interscience, New York, 1958. MR 22 \#8302.

6. D. Kleinecke, Almost-finite, compact, and inessential operators, Proc. Amer. Math. Soc. 14 (1963), 863-868. MR 27 \#5136.

7. D. Lay, Characterizations of the essential spectrum of F. E. Browder, Bull. Amer. Math. Soc. 74 (1968), 246-248. MR $36 \# 4370$.

8. W. Pfaffenberger, Operator algebras and related topics, Doctoral Dissertation, University of Oregon, Eugene, Ore., 1969.

9. C. Rickart, General the ory of Banach algebras, University Series in Higher Matho, Van Nostrand, Princeton, N. J., 1960. MR 22 \#5903.

10. A. F. Ruston, Operators with a Fredholm theory, J. London Math. Soc. 29 (1954), 318-326. MR 15, 965.

11. M. Schechter, On the essential spectrum of an arbitrary operator. I, J. Math. Anal. Appl. 13 (1966), 205-215. MR $32 \# 6230$.

12. A. E. Taylor, Introduction to functional analysis, Wiley, New York; Chapman \& Hall, London, 1958. MR 20 \#5411.

13. - Theorems on ascent, descent, nullity and defect of linear operators, Math. Ann. 163 (1966), 18-49. MR 32 \#8169.

14. T. T. West, Riesz operators in Banach spaces, Proc. London Math. Soc. (3) 16 (1966), 131-140. MR $33 \# 1742$.

DEPARTMENT OF MATHEMATICS, UNIVERSITY OF OREGON, EUGENE, OREGON 97403

Current address: Department of Mathematics, Louisiana State University, Baton Rouge, Louisiana 70803 